\title{
THE COMPACTNESS OF MINIMIZING SEQUENCES FOR A NONLINEAR SCHRÖDINGER SYSTEM WITH POTENTIALS
}

\author{
NORIHISA IKOMA AND YASUHITO MIYAMOTO
}

ABSTRACT. In this paper, we consider the following minimizing problem with two constraints:

$$
\inf \left\{E(u) \mid u=\left(u_{1}, u_{2}\right),\left\|u_{1}\right\|_{L^{2}}^{2}=\alpha_{1},\left\|u_{2}\right\|_{L^{2}}^{2}=\alpha_{2}\right\},
$$

where $\alpha_{1}, \alpha_{2}>0$ and $E(u)$ is defined by

$$
E(u):=\int_{\mathbf{R}^{N}}\left\{\frac{1}{2} \sum_{i=1}^{2}\left(\left|\nabla u_{1}\right|^{2}+V_{i}(x)\left|u_{i}\right|^{2}\right)-\sum_{i=1}^{2} \frac{\mu_{i}}{2 p_{i}+2}\left|u_{i}\right|^{2 p_{i}+2}-\frac{\beta}{p_{3}+1}\left|u_{1}\right|^{p_{3}+1}\left|u_{2}\right|^{p_{3}+1}\right\} \mathrm{d} x .
$$

Here $N \geq 1, \mu_{1}, \mu_{2}, \beta>0$ and $V_{i}(x)(i=1,2)$ are given functions. For $V_{i}(x)$, we consider two cases: (i) both of $V_{1}$ and $V_{2}$ are bounded, (ii) one of $V_{1}$ and $V_{2}$ is bounded. Under some assumptions on $V_{i}$ and $p_{j}$, we discuss the compactness of any minimizing sequence.

\section{INTRODUCTION}

In this paper, we discuss the following minimizing problem: for $\alpha=\left(\alpha_{1}, \alpha_{2}\right) \in[0, \infty)^{2}$, $(1.1)$

$$
\begin{aligned}
e(\alpha) & :=\inf \left\{E(u) \mid u=\left(u_{1}, u_{2}\right) \in M(\alpha)\right\}\left(=e\left(\alpha_{1}, \alpha_{2}\right)\right), \\
E(u) & :=\frac{1}{2} \sum_{i=1}^{2} \int_{\mathbf{R}^{N}}\left|\nabla u_{i}\right|^{2}+V_{i}(x)\left|u_{i}\right|^{2} \mathrm{~d} x-\int_{\mathbf{R}^{N}} \sum_{i=1}^{2} \frac{\mu_{i}}{2 p_{i}+2}\left|u_{i}\right|^{2 p_{i}+2}+\frac{\beta}{p_{3}+1}\left|u_{1}\right|^{p_{3}+1}\left|u_{2}\right|^{p_{3}+1} \mathrm{~d} x, \\
M(\alpha) & :=\left\{u=\left(u_{1}, u_{2}\right) \in H \mid\left\|u_{1}\right\|_{L^{2}}^{2}=\alpha_{1},\left\|u_{2}\right\|_{L^{2}}^{2}=\alpha_{2}\right\}, H:=H^{1} \times H^{1}, H^{1}:=H^{1}\left(\mathbf{R}^{N}, \mathbf{C}\right) .
\end{aligned}
$$

Here $N \geq 1,0<p_{1}, p_{2}, p_{3}<2 / N, 0<\mu_{1}, \mu_{2}, \beta$ are constants, $V_{1}(x), V_{2}(x): \mathbf{R}^{N} \rightarrow \mathbf{R}$ are given functions and $\left\|u_{i}\right\|_{L^{2}}^{2}:=\int_{\mathbf{R}^{N}}\left|u_{i}\right|^{2} \mathrm{~d} x$. Though we formally include the case $\alpha_{1}=0$ or $\alpha_{2}=0$ in (1.1), we are interested in the case $\alpha_{1}, \alpha_{2}>0$. The problem (1.1) is related to a Schrödinger system

$$
\left\{\begin{array}{l}
i \partial_{t} \psi_{1}+\Delta \psi_{1}-V_{1}(x) \psi_{1}+\left(\mu_{1}\left|\psi_{1}\right|^{2 p_{1}}+\beta\left|\psi_{1}\right|^{p_{3}-1}\left|\psi_{2}\right|^{p_{3}+1}\right) \psi_{1}=0 \\
i \partial_{t} \psi_{2}+\Delta \psi_{2}-V_{2}(x) \psi_{2}+\left(\mu_{2}\left|\psi_{2}\right|^{2 p_{2}}+\beta\left|\psi_{1}\right|^{p_{3}+1}\left|\psi_{2}\right|^{p_{3}-1}\right) \psi_{2}=0
\end{array}\right.
$$

and (1.2) appears in the field of the Bose-Einstein condensation and nonlinear optics [13,15-17,35,38]. If $u=\left(u_{1}, u_{2}\right)$ is a minimizer of (1.1), then there exist $\lambda_{1}, \lambda_{2} \in \mathbf{R}$ such that

$$
\begin{cases}-\Delta u_{1}+V_{1}(x) u_{1}-\left(\mu_{1}\left|u_{1}\right|^{2 p_{1}}+\beta\left|u_{1}\right|^{p_{3}-1}\left|u_{2}\right|^{p_{3}+1}\right) u_{1}=-\lambda_{1} u_{1} & \text { in } \mathbf{R}^{N}, \\ -\Delta u_{2}+V_{2}(x) u_{2}-\left(\mu_{2}\left|u_{2}\right|^{2 p_{2}}+\beta\left|u_{1}\right|^{p_{3}+1}\left|u_{2}\right|^{p_{3}-1}\right) u_{2}=-\lambda_{2} u_{2} & \text { in } \mathbf{R}^{N}\end{cases}
$$

Hence, $u=\left(u_{1}, u_{2}\right)$ is a standing wave solution of (1.2), that is, a solution of (1.2) of the form $\left(\psi_{1}(t, x), \psi_{2}(t, x)\right)=\left(e^{i \lambda_{1} t} u_{1}(x), e^{i \lambda_{2} t} u_{2}(x)\right)$.

Recently, the problem (1.1) and related topics are studied in various settings. Let us consider the case $V_{1}(x) \equiv 0 \equiv V_{2}(x)$ and we refer to [7,21,30,32,33,36]. In particular, Gou and Jeanjean [21] proved that any minimizing sequence for (1.1) is compact in $H$ up to translations. On the other hand, Gou [20] treated (1.1) with partial confinements when $N=3$, that is, $V_{1}\left(x_{1}, x_{2}, x_{3}\right)=V_{2}\left(x_{1}, x_{2}, x_{3}\right)=x_{1}^{2}+x_{2}^{2}$, and showed the compactness of minimizing sequence up to translations in $x_{3}$. The problem (1.1) with $V_{1}(x), V_{2}(x) \rightarrow \infty$ as $|x| \rightarrow \infty$ was also studied in [22,23,34], and in these papers, the properties of

2010 Mathematics Subject Classification. 35J50, 35J20, 35J61, 35Q55.

Key words and phrases. Minimizing problem, the multiple $L^{2}$-constraints, Nonlinear Schrödinger system, Interaction Estimates. 
minimizers were investigated. For other minimizing problems with multi-constraint conditions, we refer to $[1,2,8-12,18,24,42]$.

The aim of this paper is to treat bounded potentials, that is, we assume that at least one of $V_{1}$ and $V_{2}$ is bounded. More precisely, we consider two cases: (i) both $V_{1}$ and $V_{2}$ satisfy (V1') below, (ii) $V_{1}$ and $V_{2}$ satisfy (V2') below.

(V1') $V \in C\left(\mathbf{R}^{N}, \mathbf{R}\right) \cap L^{\infty}\left(\mathbf{R}^{N}, \mathbf{R}\right), V_{\infty}:=\lim _{|y| \rightarrow \infty} V(y)$ and $V(x) \leq V_{\infty}$ for all $x \in \mathbf{R}^{N}$.

(V2') $V_{1}$ satisfies (V1'), $V_{2} \in C\left(\mathbf{R}^{N}, \mathbf{R}\right)$ and $V_{2}(x) \rightarrow \infty$ as $|x| \rightarrow \infty$.

We note that under (V2'), $H$ and $e(\alpha)$ are replaced by

$$
\begin{aligned}
\widetilde{e}(\alpha) & :=\inf _{u \in \widetilde{M}(\alpha)} E(u), \quad \widetilde{M}(\alpha):=\widetilde{H} \cap M(\alpha), \\
\widetilde{H}_{2} & :=\left\{\left.u_{2} \in H^{1}\left(\mathbf{R}^{N}\right)\left|\int_{\mathbf{R}^{N}} V_{2}(x)\right| u_{2}\right|^{2} \mathrm{~d} x<\infty\right\}, \quad \widetilde{H}:=H^{1} \times \widetilde{H}_{2} .
\end{aligned}
$$

For exponents $p_{1}, p_{2}, p_{3}$, we introduce:

(p1) $0<p_{1}, p_{2}, p_{3}<2 / N$.

(p2) When $N \geq 5,0<p_{1}, p_{2} \leq 1 /(N-2)$.

We first consider the case where both of $V_{1}$ and $V_{2}$ are bounded:

Theorem 1.1. Assume that $V_{1}$ and $V_{2}$ satisfy $\left(\mathrm{V} 1^{\prime}\right)$, either $V_{1}(x)$ or $V_{2}(x)$ is not a constant function, (p1), (p2) and $\alpha_{i}>0(i=1,2)$. Then every minimizing sequence $\left(u_{n}\right)$ for $e(\alpha)$, namely, $\left(u_{n}\right) \subset M(\alpha)$ and $E\left(u_{n}\right) \rightarrow e(\alpha)$, has a strongly convergent subsequence in $H$. Hence, $e(\alpha)$ is attained and the set of all minimizers is compact in $H$.

Next, let us consider the case where $V_{1}$ is bounded and $V_{2}(x) \rightarrow \infty$ as $|x| \rightarrow \infty$ :

Theorem 1.2. Assume (V2'), (p1) and $\alpha_{i}>0(i=1,2)$. Then, every minimizing sequence $\left(u_{n}\right) \subset$ $\widetilde{M}(\alpha)$ for $\widetilde{e}(\alpha)$ has a strongly convergent subsequence in $\widetilde{H}$. Hence, $\widetilde{e}(\alpha)$ is attained and the set of all the minimizers is compact in $\widetilde{H}$.

Remark 1.3. (i) In Theorem 1.2, the condition (p2) is not necessary.

(ii) Even if we exchange the roles of $V_{1}(x)$ and $V_{2}(x)$, Theorem 1.2 still holds.

Finally we state one property of a set of minimizers in Theorems 1.1 and 1.2. Since this property can be proved as in Montefusco, Pellacci and Squassina [31], we omit the proof for it.

Proposition 1.4. Let $\alpha_{i}>0(i=1,2)$ and set

$$
\mathcal{M}(\alpha):=\{u \in M(\alpha) \mid E(u)=e(\alpha)\} .
$$

Then

$$
\mathcal{M}(\alpha)=\left\{\left(e^{i \theta_{1}} w_{1}, e^{i \theta_{2}} w_{2}\right) \mid 0 \leq \theta_{1}, \theta_{2} \leq 2 \pi, w_{1}(x), w_{2}(x)>0,\left(w_{1}, w_{2}\right) \in \mathcal{M}(\alpha)\right\} .
$$

The same claim holds for $\widetilde{e}(\alpha)$ :

$$
\begin{aligned}
\widetilde{\mathcal{M}}(\alpha): & =\{u \in \widetilde{M}(\alpha) \mid E(u)=\widetilde{e}(\alpha)\} \\
& =\left\{\left(e^{i \theta_{1}} w_{1}, e^{i \theta_{2}} w_{2}\right) \mid 0 \leq \theta_{1}, \theta_{2} \leq 2 \pi, w_{1}(x), w_{2}(x)>0,\left(w_{1}, w_{2}\right) \in \widetilde{\mathcal{M}}(\alpha)\right\} .
\end{aligned}
$$

Let us state differences between the known results and ours, and difficulties to prove Theorem 1.1 (here we force on Theorem 1.1). Since we deal with bounded potentials, the embedding $H \subset$ $L^{2}\left(\mathbf{R}^{N}\right) \times L^{2}\left(\mathbf{R}^{N}\right)$ is not compact. According to Lions [27,28], we notice that the compactness of any minimizing sequence for $e(\alpha)$ is equivalent to the strict subadditivity of $e(\alpha)$ : for all $\left(\theta_{1}, \theta_{2}\right) \in[0,1] \times[0,1]$ with $\left(\theta_{1}, \theta_{2}\right) \neq(1,1)$,

$$
e\left(\alpha_{1}, \alpha_{2}\right)<e\left(\theta_{1} \alpha_{1}, \theta_{2} \alpha_{2}\right)+e_{\infty}\left(\left(1-\theta_{1}\right) \alpha_{1},\left(1-\theta_{2}\right) \alpha_{2}\right)
$$


where

$$
e_{\infty}(\alpha):=\inf _{u \in M(\alpha)} E_{\infty}(u), \quad E_{\infty}(u):=\int_{\mathbf{R}^{N}} \sum_{i=1}^{2}\left(\frac{\left|\nabla u_{i}\right|^{2}}{2}-\frac{\mu_{i}}{2 p_{i}+2}\left|u_{i}\right|^{2 p_{i}+2}\right)-\frac{\beta}{p_{3}+1}\left|u_{1} u_{2}\right|^{p_{3}+1} \mathrm{~d} x .
$$

Here we note that when $p_{3} \geq 1$, by following the argument in [28, p.228-p.229] (see also [10,14] and Appendix A), it is possible to prove (1.4) under the assumption $V_{1}(x), V_{2}(x) \leq 0$ and $V_{1}(x), V_{2}(x) \rightarrow 0$ as $|x| \rightarrow \infty$. However, when $0<p_{3}<1$, it is not straightforward to exploit the argument in [28] and we face a difficulty in proving (1.4).

In the cases $V_{1}(x) \equiv 0 \equiv V_{2}(x)$ [21] and $V_{1}\left(x_{1}, x_{2}, x_{3}\right)=x_{1}^{2}+x_{2}^{2}=V_{2}\left(x_{1}, x_{2}, x_{3}\right)$ [20], to get (1.4), they use the coupled rearrangement due to Shibata [41]. However, in our setting, this technique is not applicable since we do not suppose any symmetry of $V_{1}(x)$ and $V_{2}(x)$.

In this paper, we show (1.4) by combining the scheme in $[24,40]$ and the interaction estimate in Bahri-Li [3]. This approach is also taken in [25] for a minimizing problem corresponding to a scalar equation with potential and general nonlinearity. Compared to the scalar case [25], we have the following differences. First, we need to observe the decay estimate of nonnegative solutions to (1.3). This will be discussed in subsection 3.1 and we point out that the decay properties of solutions to (1.3) depend on the size of $p_{3}, \lambda_{1}$ and $\lambda_{2}$. Next, we need to prepare some inequality which is used in the interaction estimate for the term $\int_{\mathbf{R}^{N}} u_{1}^{p_{3}+1} u_{2}^{p_{3}+1} \mathrm{~d} x$. We emphasize that when $0<p_{3}<1$, the term $u_{1}^{p_{3}+1} u_{2}^{p_{3}+1}$ is not of class $C^{2}$, hence, we need a careful analysis. See Lemma 3.4 (ii).

This paper is organized as follows. In section 2, we do some preparations which are necessary for proofs of Theorems 1.1 and 1.2. In section 3, we observe the decay properties of solutions of (1.3) and prove useful inequalities and Theorem 1.1. Section 4 is devoted to a proof of Theorem 1.2 and in Appendix A, we provide a proof of $(1.4)$ when $p_{3} \geq 1$ by following the arguments in [28] (cf. [10,14]).

\section{Preliminaries}

In this section, we shall prove several results which are necessary for the proofs of Theorems 1.1 and 1.2. Similar results are used in $[24,25]$.

We first notice that for every $b_{1}, b_{2} \in \mathbf{R}$, the minimizing problem for $e(\alpha)$ (resp. $\widetilde{e}(\alpha)$ ) is equivalent to

$$
\begin{aligned}
& \inf \left\{E(u)-\sum_{i=1}^{2} \frac{b_{i}}{2}\left\|u_{i}\right\|_{L^{2}}^{2} \mid u \in M(\alpha)\right\}=e(\alpha)-\sum_{i=1}^{2} \frac{b_{i}}{2} \alpha_{i}, \\
& \text { (resp. } \left.\inf \left\{E(u)-\sum_{i=1}^{2} \frac{b_{i}}{2}\left\|u_{i}\right\|_{L^{2}}^{2} \mid u \in \widetilde{M}(\alpha)\right\}=\widetilde{e}(\alpha)-\sum_{i=1}^{2} \frac{b_{i}}{2} \alpha_{i}\right) .
\end{aligned}
$$

Therefore, without loss of generality, we may assume the following conditions instead of (V1') and (V2'):

(V1) $V \in C\left(\mathbf{R}^{N}, \mathbf{R}\right), V(x) \leq 0$ for all $x \in \mathbf{R}^{N}$ and $\lim _{|x| \rightarrow \infty} V(x)=0$.

(V2) $V_{1}$ satisfies (V1), $V_{2} \in C\left(\mathbf{R}^{N}, \mathbf{R}\right), 1=\inf _{x \in \mathbf{R}^{N}} V_{2}(x)$ and $V_{2}(x) \rightarrow \infty$ as $|x| \rightarrow \infty$.

We set

$$
\left\langle u_{i}, v_{i}\right\rangle_{V_{i}}:=\operatorname{Re} \int_{\mathbf{R}^{N}} \nabla u_{i} \cdot \overline{\nabla v_{i}} \mathrm{~d} x+\operatorname{Re} \int_{\mathbf{R}^{N}} V_{i}(x) u_{i} \overline{v_{i}} \mathrm{~d} x, \quad\left\|u_{i}\right\|_{V_{i}}^{2}:=\left\langle u_{i}, u_{i}\right\rangle_{V_{i}} .
$$

Even though the quantity $\|\cdot\|_{V_{i}}$ may not be a norm on $H^{1}$, this notation will be convenient in subsection 3.3. Remark that under $(\mathrm{V} 2),\|\cdot\|_{V_{2}}$ is a norm on $\widetilde{H}_{2}$ and $\left(\widetilde{H}_{2},\langle\cdot, \cdot\rangle_{V_{2}}\right)$ is a Hilbert space over $\mathbf{R}$.

To prove Theorem 1.1, we begin with the following lemma:

Lemma 2.1. Assume (p1).

(i) Suppose (V1). Then $-\infty<e(\alpha)$ holds and every minimizing sequence for $e(\alpha)$ is bounded in $H$. If we assume (V2) instead of (V1), then $-\infty<\widetilde{e}(\alpha)$ and any minimizing sequence for $\widetilde{e}(\alpha)$ is bounded in $\widetilde{H}$. 
(ii) Under (V1), e $(\alpha)<0$ holds .

(iii) Suppose (V1). Then $e(\alpha) \leq e(\gamma)+e_{\infty}(\alpha-\gamma)$ for any $\gamma=\left(\gamma_{1}, \gamma_{2}\right) \in\left[0, \alpha_{1}\right] \times\left[0, \alpha_{2}\right]$ where

$$
\begin{aligned}
& e_{\infty}(\alpha):=\inf \left\{E_{\infty}(u) \mid u \in M(\alpha)\right\}, \\
& E_{\infty}(u):=\frac{1}{2} \int_{\mathbf{R}^{N}} \sum_{i=1}^{2}\left|\nabla u_{i}\right|^{2} \mathrm{~d} x-\int_{\mathbf{R}^{N}} \frac{\mu_{1}\left|u_{1}\right|^{2 p_{1}+2}}{2 p_{1}+2}+\frac{\mu_{2}\left|u_{2}\right|^{2 p_{2}+2}}{2 p_{2}+2}+\frac{\beta\left|u_{1}\right|^{p_{3}+1}\left|u_{2}\right|^{p_{3}+1}}{p_{3}+1} \mathrm{~d} x .
\end{aligned}
$$

If we suppose (V2) instead of (V1), then $\widetilde{e}(\alpha) \leq \widetilde{e}\left(\alpha_{1}-\gamma, \alpha_{2}\right)+e_{1}(\gamma)$ for all $\gamma \in\left[0, \alpha_{1}\right]$ where

$$
\begin{aligned}
e_{1}(\gamma) & :=\inf \left\{E_{1}\left(u_{1}\right) \mid u_{1} \in H^{1}\left(\mathbf{R}^{N}\right),\left\|u_{1}\right\|_{L^{2}}^{2}=\gamma\right\}, \\
E_{1}\left(u_{1}\right) & :=\frac{1}{2} \int_{\mathbf{R}^{N}}\left|\nabla u_{1}\right|^{2} \mathrm{~d} x-\frac{\mu_{2}}{2 p_{2}+2} \int_{\mathbf{R}^{N}}\left|u_{1}\right|^{2 p_{2}+2} \mathrm{~d} x .
\end{aligned}
$$

Proof. (i) We first prove the assertion for $e(\alpha)$. Let $\left(u_{n}\right) \subset M(\alpha)$ satisfy $E\left(u_{n}\right) \rightarrow e(\alpha)$. It suffices to show that $\left(\nabla u_{n, i}\right)(i=1,2)$ are bounded in $L^{2}\left(\mathbf{R}^{N}\right)$. By Gagliardo-Nirenberg's inequality,

$$
\|u\|_{L^{2 p+2}}^{2 p+2} \leq C\|\nabla u\|_{L^{2}}^{\theta(2 p+2)}\|u\|_{L^{2}}^{(1-\theta)(2 p+2)}, \quad \theta:=\left(\frac{1}{2}-\frac{1}{2 p+2}\right) N .
$$

Thus if $p<2 / N$, then one sees that $(2 p+2) \theta=N p<2$. Since $V_{i}(x) \leq 0$ and

$$
\begin{aligned}
E\left(u_{n}\right) \geq & \frac{1}{2}\left(\left\|\nabla u_{n, 1}\right\|_{L^{2}}^{2}+\left\|\nabla u_{n, 2}\right\|_{L^{2}}^{2}\right)+\frac{1}{2} \int_{\mathbf{R}^{N}} V_{1}(x)\left|u_{n, 1}\right|^{2}+V_{2}(x)\left|u_{n, 2}\right|^{2} \mathrm{~d} x \\
& \quad \frac{\mu_{1}\left\|u_{n, 1}\right\|_{L^{2 p_{1}+2}}^{2 p_{1}+2}}{2 p_{1}+2}-\frac{\mu_{2}\left\|u_{n, 2}\right\|_{L^{2 p_{2}+2}}^{2 p_{2}+2}}{2 p_{2}+2}-\frac{\beta\left\|u_{n, 1}\right\|_{L^{2 p_{3}+2}}^{p_{3}+1}\left\|u_{n, 2}\right\|_{L^{2 p_{3}+2}}^{p_{3}+1}}{p_{3}+1} \\
\geq & \frac{1}{2} \sum_{i=1}^{2}\left\|\nabla u_{n, i}\right\|_{L^{2}}^{2}-C\left(\left\|\nabla u_{n, 1}\right\|_{L^{2}}^{N p_{1}}+\left\|\nabla u_{n, 2}\right\|_{L^{2}}^{N p_{2}}+\left\|\nabla u_{n, 1}\right\|_{L^{2}}^{N p_{3} / 2}\left\|\nabla u_{n, 2}\right\|_{L^{2}}^{N p_{3} / 2}\right),
\end{aligned}
$$

we have $e(\alpha)>-\infty$ and $\left(\nabla u_{n, i}\right)(i=1,2)$ are bounded in $L^{2}\left(\mathbf{R}^{N}\right)$.

On the other hand, when (V2) holds, as in the above, we see that $\left(\left\|\nabla u_{n, i}\right\|_{L^{2}}\right)(i=1,2)$ are bounded. Furthermore, from (2.2), it follows that $\sup _{n \geq 1} \int_{\mathbf{R}^{N}} V_{2}(x)\left|u_{n, 2}\right|^{2} \mathrm{~d} x<\infty$, hence, $\left(u_{n}\right)$ is bounded in $\widetilde{H}$.

(ii) By (V1), for any $u \in M(\alpha)$, it is immediate to check $E(u) \leq E_{\infty}(u)$, which yields $e(\alpha) \leq e_{\infty}(\alpha)$. According to [21], it is known that $e_{\infty}(\alpha)<0$. Hence, we have $e(\alpha)<0$.

(iii) Since we may prove both of inequalities in a similar way, we only show the desired inequality for $e(\alpha)$. For every $\varepsilon>0$ and $\gamma \in\left[0, \alpha_{1}\right] \times\left[0, \alpha_{2}\right]$, we may find $\varphi_{\varepsilon}, \psi_{\varepsilon} \in\left(C_{0}^{\infty}\left(\mathbf{R}^{N}\right)\right)^{2}$ so that

$$
\varphi_{\varepsilon} \in M(\gamma), \psi_{\varepsilon} \in M(\alpha-\gamma), E\left(\varphi_{\varepsilon}\right) \leq e(\gamma)+\varepsilon, E_{\infty}\left(\psi_{\varepsilon}\right) \leq e_{\infty}(\alpha-\gamma)+\varepsilon .
$$

Set $u_{\varepsilon, n}(x):=\varphi_{\varepsilon}(x)+\psi_{\varepsilon}\left(x-n \mathbf{e}_{1}\right)$. Since $\varphi_{\varepsilon}, \psi_{\varepsilon}$ have compact support, we have $u_{\varepsilon, n} \in M(\alpha)$ for sufficiently large $n$ and $e(\alpha) \leq E\left(u_{\varepsilon, n}\right)=E\left(\varphi_{\varepsilon}\right)+E\left(\psi_{\varepsilon}\left(\cdot-n \mathbf{e}_{1}\right)\right)$. By letting $n \rightarrow \infty$ and noting $E_{\infty}(u(\cdot-y))=E_{\infty}(u)$ for all $y \in \mathbf{R}^{N},(\mathrm{~V} 1)$ gives

$$
e(\alpha) \leq E\left(\varphi_{\varepsilon}\right)+E\left(\psi_{\varepsilon}\left(\cdot-n \mathbf{e}_{1}\right)\right) \leq E\left(\varphi_{\varepsilon}\right)+E_{\infty}\left(\psi_{\varepsilon}\left(\cdot-n \mathbf{e}_{1}\right)\right) \leq e(\gamma)+e_{\infty}(\alpha-\gamma)+2 \varepsilon .
$$

Since $\varepsilon>0$ is arbitrary, the desired inequality for $e(\alpha)$ holds.

Next, we show that it is enough to consider a minimizing sequence whose components are nonnegative.

Lemma 2.2. Under (p1) and (V1), let $\left(u_{n}\right) \subset M(\alpha)$ satisfy $E\left(u_{n}\right) \rightarrow e(\alpha)$. Then $\left|u_{n}\right|(x):=$ $\left(\left|u_{n, 1}(x)\right|,\left|u_{n, 2}(x)\right|\right)$ is also a minimizing sequence. Moreover, if $\left(\left|u_{n}\right|\right)$ has a strongly convergent subsequence in $H$, so is $\left(u_{n}\right)$.

The same assertions hold when we suppose (V2) instead of (V1).

Proof. For $e(\alpha)$, by [26, Theorem 6.17], we have $\left\|\nabla\left|u_{n, i}\right|\right\|_{L^{2}}^{2} \leq\left\|\nabla u_{n, i}\right\|_{L^{2}}^{2}$. Since $\left|u_{n}\right| \in M(\alpha)$, one observes that $E\left(\left|u_{n}\right|\right) \leq E\left(u_{n}\right)$. Hence, $\left(\left|u_{n}\right|\right)$ is also a minimizing sequence.

Next, assume that $\left(\left|u_{n}\right|\right)$ has a strongly convergent subsequence in $H$. Hereafter, we still write $\left(u_{n}\right)$ even after taking a subsequence. Thus, we suppose that $\left|u_{n}\right| \rightarrow\left(v_{1}, v_{2}\right)$ strongly in $H$. Since $\left(u_{n}\right)$ is 
bounded in $H$ by Lemma 2.1 , we have $u_{n} \rightarrow u_{0}=\left(u_{0,1}, u_{0,2}\right)$ weakly in $H$ and $u_{n, i} \rightarrow u_{0, i}$ in $L_{\text {loc }}^{q}\left(\mathbf{R}^{N}\right)$ for $1 \leq q<2^{*}=2 N /(N-2)$. Therefore, we infer that $v_{i}(x)=\left|u_{0, i}(x)\right|$.

From $\left|u_{n, i}\right| \rightarrow\left|u_{0, i}\right|$ strongly in $L^{2}\left(\mathbf{R}^{N}\right)$ with $i=1,2$, after taking a sequence, we may find $w_{i} \in$ $L^{2}\left(\mathbf{R}^{N}\right)$ such that $\left|u_{n, i}(x)\right| \leq w_{i}(x)$ and $\left|u_{n, i}(x)\right| \rightarrow\left|u_{0, i}(x)\right|$ for a.a. $x \in \mathbf{R}^{N}$. The dominated convergence theorem yields $u_{n} \rightarrow u_{0}$ strongly in $L^{2}\left(\mathbf{R}^{N}\right) \times L^{2}\left(\mathbf{R}^{N}\right)$ and $u_{n} \rightarrow u_{0}$ strongly in $L^{q}\left(\mathbf{R}^{N}\right) \times$ $L^{q}\left(\mathbf{R}^{N}\right)$ for every $2 \leq q<2^{*}$. In particular, $u_{0} \in M(\alpha)$.

Finally, by the weak lower semicontinuity and the facts $u_{0} \in M(\alpha)$ and $u_{n} \rightarrow u_{0}$ in $L^{q}\left(\mathbf{R}^{N}\right) \times L^{q}\left(\mathbf{R}^{N}\right)$ for $2 \leq q<2^{*}$, we obtain

$$
e(\alpha) \leq E\left(u_{0}\right) \leq \liminf _{n \rightarrow \infty} E\left(u_{n}\right)=e(\alpha),
$$

which implies $\left\|\nabla u_{n, i}\right\|_{L^{2}}^{2} \rightarrow\left\|\nabla u_{0, i}\right\|_{L^{2}}^{2}$. Hence, by recalling $\nabla u_{n, i} \rightarrow \nabla u_{0, i}$ weakly in $L^{2}\left(\mathbf{R}^{N}\right)$, one sees that $\nabla u_{n, i} \rightarrow \nabla u_{0, i}$ strongly in $L^{2}\left(\mathbf{R}^{N}\right)$. Thus $u_{n} \rightarrow u_{0}$ strongly in $H$.

On the other hand, for $\widetilde{e}(\alpha)$, as in the above, we may prove $u_{n} \rightarrow u_{0}$ strongly in $L^{q}\left(\mathbf{R}^{N}\right) \times L^{q}\left(\mathbf{R}^{N}\right)$ for $2 \leq q<2^{*}$. Thus,

$$
\widetilde{e}(\alpha) \leq E\left(u_{0}\right) \leq \liminf _{n \rightarrow \infty} E\left(u_{n}\right)=\widetilde{e}(\alpha)
$$

and this yields $\left\|u_{n, 2}\right\|_{V_{2}}^{2} \rightarrow\left\|u_{0,2}\right\|_{V_{2}}^{2}$. Hence, $u_{n} \rightarrow u_{0}$ strongly in $\widetilde{H}$.

Next, we show the existence of Palais-Smale sequence close to any given minimizing sequence.

Lemma 2.3. $\quad$ (i) Suppose (p1) and (V1), and let $\left(u_{n}\right) \subset M(\alpha)$ be a minimizing sequence. Then there exist $\left(v_{n}\right) \subset M(\alpha)$ and $\left(\lambda_{n, i}\right) \subset \mathbf{R}$ such that $\left(\lambda_{n, i}\right)$ are bounded and

$$
\left\|u_{n}-v_{n}\right\|_{H} \rightarrow 0, \quad E^{\prime}\left(v_{n}\right)+\lambda_{n, 1} Q_{1}^{\prime}\left(v_{n}\right)+\lambda_{n, 2} Q_{2}^{\prime}\left(v_{n}\right) \rightarrow 0 \quad \text { strongly in } H^{*}
$$

where

$$
\begin{aligned}
E^{\prime}(u) v= & \operatorname{Re} \int_{\mathbf{R}^{N}} \sum_{i=1}^{2} \nabla u_{i} \cdot \overline{\nabla v_{i}}+V_{i}(x) u_{i} \overline{v_{i}} \mathrm{~d} x-\operatorname{Re} \int_{\mathbf{R}^{N}} \mu_{1}\left|u_{1}\right|^{2 p_{1}} u_{1} \overline{v_{1}}+\mu_{2}\left|u_{2}\right|^{2 p_{2}} u_{2} \overline{v_{2}} \mathrm{~d} x \\
& -\operatorname{Re} \int_{\mathbf{R}^{N}} \beta\left|u_{1}\right|^{p_{3}-1}\left|u_{2}\right|^{p_{3}+1} u_{1} \overline{v_{1}}+\beta\left|u_{1}\right|^{p_{3}+1}\left|u_{2}\right|^{p_{3}-1} u_{2} \overline{v_{2}} \mathrm{~d} x, \\
Q_{i}(u):= & \frac{1}{2} \int_{\mathbf{R}^{N}}\left|u_{i}\right|^{2} \mathrm{~d} x, \quad Q_{i}^{\prime}(u) v=\operatorname{Re} \int_{\mathbf{R}^{N}} u_{i} \overline{v_{i}} \mathrm{~d} x .
\end{aligned}
$$

(ii) Assume (p1) and (V2). Then for every minimizing sequence $\left(u_{n}\right) \subset \widetilde{M}(\alpha)$ for $\widetilde{e}(\alpha)$, there exists $\left(v_{n}\right) \subset \widetilde{M}(\alpha)$ and $\left(\lambda_{n, i}\right) \subset \mathbf{R}$ such that $\left(\lambda_{n, i}\right)$ are bounded and

$$
\left\|u_{n}-v_{n}\right\|_{H}+\left\|u_{n, 1}-v_{n, 1}\right\|_{V_{1}} \rightarrow 0, \quad E^{\prime}\left(v_{n}\right)+\lambda_{n, 1} Q_{1}^{\prime}\left(v_{n}\right)+\lambda_{n, 2} Q_{2}^{\prime}\left(v_{n}\right) \rightarrow 0 \quad \text { strongly in }(\widetilde{H})^{*} .
$$

Proof. Since a proof for $\widetilde{e}(\alpha)$ is similar to the one for $e(\alpha)$, we only show (i). Note that $M(\alpha)$ is a closed set in $H$. Applying Ekeland's variational principle for $E$ and $\left(u_{n}\right)$ on $M(\alpha)$ ( [29, Theorem 4.1 and Remark 4.1]), we may find $\left(v_{n}\right) \subset M(\alpha)$ such that for $\varepsilon_{n}:=E\left(u_{n}\right)-e(\alpha) \geq 0$,

$$
E\left(v_{n}\right) \leq E\left(u_{n}\right), \quad\left\|u_{n}-v_{n}\right\|_{H} \leq \sqrt{\varepsilon_{n}}, \quad E\left(v_{n}\right) \leq E(w)+\sqrt{\varepsilon_{n}}\left\|v_{n}-w\right\|_{H} \quad \text { for all } w \in M(\alpha) .
$$

Thus, $\left(v_{n}\right)$ is also a minimizing sequence.

Since

$$
M(\alpha)=\left\{u \in H \mid Q_{1}(u)=\frac{\alpha_{1}}{2}, Q_{2}(u)=\frac{\alpha_{2}}{2}\right\}, \quad Q_{1}^{\prime}(u)\left[\left(u_{1}, 0\right)\right]=\alpha_{1}, \quad Q_{2}^{\prime}(u)\left[\left(0, u_{2}\right)\right]=\alpha_{2},
$$

we see that $Q_{1}^{\prime}(u)$ and $Q_{2}^{\prime}(u)$ are linearly independent for each $u \in M(\alpha)$, and that $M(\alpha)$ is a Hilbert manifold with codimension 2 . Its tangent space at $u \in M(\alpha)$ is given by

$$
T_{u} M(\alpha)=\left\{v \in H \mid Q_{1}^{\prime}(u) v=0=Q_{2}^{\prime}(u) v\right\}\left(=\operatorname{span}\left\{\nabla Q_{1}(u), \nabla Q_{2}(u)\right\}^{\perp}\right)
$$

where $\nabla Q_{i}(u) \in H$ satisfies $\left\langle\nabla Q_{i}(u), v\right\rangle_{H}=Q_{i}^{\prime}(u) v$ for all $v \in H$. By $(2.3)$, one gets

$$
\left\|E^{\prime}\left(v_{n}\right)\right\|_{\left(T_{v_{n}} M(\alpha)\right)^{*}}=\sup _{w \in T_{v_{n}} M(\alpha),\|w\|_{H} \leq 1} E^{\prime}\left(v_{n}\right) w \rightarrow 0 .
$$


From (2.5), for any $\lambda_{1}, \lambda_{2} \in \mathbf{R}$, we have

$$
\left(E^{\prime}\left(v_{n}\right)+\lambda_{1} Q_{1}^{\prime}\left(v_{n}\right)+\lambda_{2} Q_{2}^{\prime}\left(v_{n}\right)\right) w=E^{\prime}\left(v_{n}\right) w \text { for all } w \in T_{v_{n}} M(\alpha) .
$$

In addition, since $\left(v_{n}\right)$ is bounded in $H$ due to Lemma 2.1, (2.4) implies that there exists $0<c_{1}<c_{2}<$ $\infty$ such that $c_{1} \leq\left\|Q_{i}^{\prime}\left(v_{n}\right)\right\|_{H^{*}} \leq c_{2}$ for every $n \geq 1$. Thus, setting $\lambda_{n, i}:=-E^{\prime}\left(v_{n}\right) \nabla Q_{i}\left(v_{n}\right) /\left\|Q_{i}^{\prime}\left(v_{n}\right)\right\|_{H^{*}}^{2}$, we observe that $\left(\lambda_{n, i}\right)$ are bounded and

$$
E^{\prime}\left(v_{n}\right)+\lambda_{n, 1} Q_{1}^{\prime}\left(v_{n}\right)+\lambda_{n, 2} Q_{2}^{\prime}\left(v_{n}\right) \rightarrow 0 \text { strongly in } H^{*} .
$$

Thus, Lemma 2.3 holds.

\section{Proof of Theorem 1.1}

In this section, we aim to prove Theorem 1.1. Before proceeding to the proof of Theorem 1.1, we prepare several useful lemmas. Throughout this section, we always assume that $V_{1}$ and $V_{2}$ satisfy (V1) and (p1) holds

3.1. Decay estimates. In this subsection, we observe the decay rate of nonnegative solutions to

$$
\begin{cases}-\Delta u_{1}+\left(V_{1}(x)+\lambda_{0,1}\right) u_{1}=\mu_{1} u_{1}^{2 p_{1}+1}+\beta u_{1}^{p_{3}} u_{2}^{p_{3}+1} & \text { in } \mathbf{R}^{N}, \\ -\Delta u_{2}+\left(V_{2}(x)+\lambda_{0,2}\right) u_{2}=\beta u_{1}^{p_{3}+1} u_{2}^{p_{3}}+\mu_{2} u_{2}^{2 p_{2}+1} & \text { in } \mathbf{R}^{N}\end{cases}
$$

where $\lambda_{0,1}, \lambda_{0,2}$ are constants satisfying

$$
0<\lambda_{0,1} \leq \lambda_{0,2}
$$

The aim of this subsection is to prove the following decay estimates:

Lemma 3.1. Let $u=\left(u_{1}, u_{2}\right)$ be a solution of (3.1) with $u_{1}(x), u_{2}(x) \geq 0$ in $\mathbf{R}^{N}$.

(i) If $u_{1} \not \equiv 0$, then for every $\lambda \in\left(0, \lambda_{0,1}\right)$, there exist $c_{0}>0$, which is independent of $\lambda$, and $C_{\lambda}>0$ such that

$$
c_{0}(1+|x|)^{-(N-1) / 2} \exp \left(-\sqrt{\lambda_{0,1}}|x|\right) \leq u_{1}(x) \leq C_{\lambda} \exp (-\sqrt{\lambda}|x|) \quad \text { for all } x \in \mathbf{R}^{N} .
$$

(ii) If $u_{1} \equiv 0$ and $u_{2} \not \equiv 0$, then for every $\lambda \in\left(0, \lambda_{0,2}\right)$, there exist $c_{0}>0$, which is independent of $\lambda$, and $C_{\lambda}>0$ such that

$$
c_{0}(1+|x|)^{-(N-1) / 2} \exp \left(-\sqrt{\lambda_{0,2}}|x|\right) \leq u_{2}(x) \leq C_{\lambda} \exp (-\sqrt{\lambda}|x|) \quad \text { for all } x \in \mathbf{R}^{N} .
$$

(iii) Suppose that $u_{1}, u_{2} \not \equiv 0$. When either $p_{3} \geq 1$ or else $0<p_{3}<1$ and $\lambda_{0,3}:=\left(1+p_{3}\right)^{2} \lambda_{0,1} /(1-$ $\left.p_{3}\right)^{2}>\lambda_{0,2}$, for every $\lambda \in\left(0, \lambda_{0,2}\right)$, there exist $c_{0}>0$, which is independent of $\lambda$, and $C_{\lambda}>0$ such that

$$
c_{0}(1+|x|)^{-(N-1) / 2} \exp \left(-\sqrt{\lambda_{0,2}}|x|\right) \leq u_{2}(x) \leq C_{\lambda} \exp (-\sqrt{\lambda}|x|) \quad \text { for all } x \in \mathbf{R}^{N} .
$$

On the other hand, when $0<p_{3}<1$ and $\lambda_{0,3} \leq \lambda_{0,2}$, for each $\lambda_{1} \in\left(0, \lambda_{0,3}\right)$ and $\lambda_{2} \in$ $\left(\lambda_{0,3}, \infty\right)$, there exist $C_{\lambda_{1}}, C_{\lambda_{2}}>0$ such that

$$
C_{\lambda_{2}} \exp \left(-\sqrt{\lambda_{2}}|x|\right) \leq u_{2}(x) \leq C_{\lambda_{1}} \exp \left(-\sqrt{\lambda_{1}}|x|\right) \quad \text { for all } x \in \mathbf{R}^{N} .
$$

Remark 3.2. Putting $V_{1}(x) \equiv 0 \equiv V_{2}(x)$ in (3.1), we observe that the same statements in Lemma 3.1 hold for solutions $w=\left(w_{1}, w_{2}\right)$ of $(3.3)$ :

$$
\begin{cases}-\Delta w_{1}+\lambda_{0,1} w_{1}=\mu_{1} w_{1}^{2 p_{1}+1}+\beta w_{1}^{p_{3}} w_{2}^{p_{3}+1} & \text { in } \mathbf{R}^{N}, \\ -\Delta w_{2}+\lambda_{0,2} w_{2}=\beta w_{1}^{p_{3}+1} w_{2}^{p_{3}}+\mu_{2} w_{2}^{2 p_{2}+1} & \text { in } \mathbf{R}^{N} .\end{cases}
$$

To show Lemma 3.1, we prepare one lemma which is a variant of [3, Proposition 1.2] and [4, Lemma II.2] (cf. [19]) and also useful for the interaction estimate below: 
Lemma 3.3. Let $0 \leq f(x), g(x)$ satisfy $f, g \in C\left(\mathbf{R}^{N} \backslash\{0\}\right) \cap L^{1}\left(\mathbf{R}^{N}\right)$ and

$$
\lim _{|x| \rightarrow \infty}(1+|x|)^{\alpha} e^{\beta|x|} g(x)=\gamma \in[0, \infty), \quad f(x) \leq C \exp (-(\beta+\eta)|x|) \quad \text { for each }|x| \geq 1
$$

for some $\alpha \geq 0, \beta \geq 0$ and $\eta>0$. Then

$$
\lim _{r \rightarrow \infty}(1+r)^{\alpha} e^{\beta r} \int_{\mathbf{R}^{N}} g(r \omega-y) f(y) \mathrm{d} y=\gamma \int_{\mathbf{R}^{N}} f(y) e^{\beta \omega \cdot y} \mathrm{~d} y \quad \text { uniformly with respect to } \omega \in \mathbf{S}^{N-1} .
$$

Proof. From the assumptions, for every $\varepsilon>0$, choose an $R_{\varepsilon}>0$ so that

$$
|x| \geq R_{\varepsilon} \quad \Rightarrow \quad(1+|x|)^{\alpha} e^{\beta|x|} g(x)-\gamma \mid \leq \varepsilon .
$$

We divide the integral into two parts:

$$
\begin{aligned}
(1+r)^{\alpha} e^{\beta r} \int_{\mathbf{R}^{N}} g(r \omega-y) f(y) \mathrm{d} y & =\left(\int_{|r \omega-y| \leq R_{\varepsilon}}+\int_{|r \omega-y|>R_{\varepsilon}}\right)(1+r)^{\alpha} e^{\beta r} g(r \omega-y) f(y) \mathrm{d} y \\
& =: J_{1}(r, \omega)+J_{2}(r, \omega) .
\end{aligned}
$$

We first show that

$$
\lim _{r \rightarrow \infty} \sup _{\omega \in \mathbf{S}^{N-1}}\left|J_{1}(r, \omega)\right|=0 .
$$

It follows from the assumptions that for $r \geq R_{\varepsilon}+1$,

$$
\begin{aligned}
\left|J_{1}(r, \omega)\right| & \leq \int_{|z| \leq R_{\varepsilon}} g(z) f(r \omega-z) e^{\beta r}(1+r)^{\alpha} \mathrm{d} z \\
& \leq \int_{|z| \leq R_{\varepsilon}} g(z) C \exp (-(\beta+\eta)|r \omega-z|) e^{\beta r}(1+r)^{\alpha} \mathrm{d} z \\
& =C \int_{|z| \leq R_{\varepsilon}} g(z) \exp (\beta\{r-|r \omega-z|\})(1+r)^{\alpha} e^{-\eta|r \omega-z|} \mathrm{d} z .
\end{aligned}
$$

Noting $r-|r \omega-z| \leq|z|, g \in L^{1}\left(B_{R_{\varepsilon}}\right)$ and

$$
\lim _{r \rightarrow \infty} \sup _{\omega \in \mathbf{S}^{N-1},|z| \leq R_{\varepsilon}}(1+r)^{\alpha} e^{-\eta|r \omega-z|}=0,
$$

we observe that (3.5) holds.

Next, we consider $J_{2}(r, \omega)$. Since

$$
J_{2}(r, \omega)=\int_{|r \omega-y|>R_{\varepsilon}} g(r \omega-y)(1+|r \omega-y|)^{\alpha} e^{\beta|r \omega-y|} f(y) e^{\beta(r-|r \omega-y|)}\left(\frac{1+r}{1+|r \omega-y|}\right)^{\alpha} \mathrm{d} y
$$

and $r-|r \omega-y| \leq|y|$, we see from (3.4) and the assumption on $f$ that

$$
\begin{aligned}
& \sup _{\omega \in \mathbf{S}^{N-1}}\left|J_{2}(r, \omega)-\gamma \int_{|r \omega-y|>R_{\varepsilon}} f(y) e^{\beta(r-|r \omega-y|)}\left(\frac{1+r}{1+|r \omega-y|}\right)^{\alpha} \mathrm{d} y\right| \\
\leq \varepsilon & \sup _{\omega \in \mathbf{S}^{N-1}} \int_{|r \omega-y|>R_{\varepsilon}} f(y) e^{\beta(r-|r \omega-y|)}\left(\frac{1+r}{1+|r \omega-y|}\right)^{\alpha} \mathrm{d} y \\
\leq & \varepsilon \sup _{\omega \in \mathbf{S}^{N-1}} \int_{\mathbf{R}^{N}} f(y) e^{\beta|y|}\left(\frac{1+|r \omega-y|+|y|}{1+|r \omega-y|}\right)^{\alpha} \mathrm{d} y \\
\leq & \varepsilon \int_{\mathbf{R}^{N}} f(y) e^{\beta|y|}(1+|y|)^{\alpha} \mathrm{d} y=C(f) \varepsilon .
\end{aligned}
$$

Next, by

$$
\begin{aligned}
\beta(r-|r \omega-y|)=\beta r\left[1-\left(1-2 \frac{\omega \cdot y}{r}+\frac{|y|^{2}}{r^{2}}\right)^{1 / 2}\right] & =\beta r\left[\frac{\omega \cdot y}{r}+O\left(\frac{|y|^{2}}{r^{2}}\right)\right] \\
& =\beta \omega \cdot y+O\left(\frac{|y|^{2}}{r}\right)
\end{aligned}
$$


we can choose a $\delta_{\varepsilon}>0$ so that

$$
|y| \leq \delta_{\varepsilon} \sqrt{r} \Rightarrow \sup _{\omega \in \mathbf{S}^{N-1}}\left|e^{\beta(r-|r \omega-y|)}-e^{\beta \omega \cdot y}\right|<\varepsilon \sup _{\omega \in \mathbf{S}^{N-1}} e^{\beta \omega \cdot y}=\varepsilon e^{\beta|y|} .
$$

Set

$$
\left[|r \omega-y|>R_{\varepsilon}\right] \cap\left[|y| \leq \delta_{\varepsilon} \sqrt{r}\right]:=I_{1}(r, \omega), \quad\left[|r \omega-y|>R_{\varepsilon}\right] \cap\left[|y|>\delta_{\varepsilon} \sqrt{r}\right]:=I_{2}(r, \omega) .
$$

Noting

we obtain

$$
\begin{aligned}
& \lim _{r \rightarrow \infty} \sup _{\omega \in \mathbf{S}^{N-1}, y \in I_{1}(r, \omega)}\left(\frac{1+r}{1+|r \omega-y|}\right)^{\alpha}=1, \\
& \lim _{r \rightarrow \infty} \sup _{\omega \in \mathbf{S}^{N-1}} \int_{\left[|y|>\delta_{\varepsilon} \sqrt{r}\right] \cup\left[|r \omega-y| \leq R_{\varepsilon}\right]} f(y) e^{\beta|y|}(1+|y|)^{\alpha} \mathrm{d} y=0,
\end{aligned}
$$

$$
\begin{aligned}
& \limsup _{r \rightarrow \infty} \sup _{\omega \in \mathbf{S}^{N-1}}\left|\int_{I_{1}(r, \omega)} f(y) e^{\beta(r-|r \omega-y|)}\left(\frac{1+r}{1+|r \omega-y|}\right)^{\alpha} \mathrm{d} y-\int_{\mathbf{R}^{N}} f(y) e^{\beta \omega \cdot y} \mathrm{~d} y\right| \\
= & \limsup _{r \rightarrow \infty} \sup _{\omega \in \mathbf{S}^{N-1}}\left|\int_{I_{1}(r, \omega)} f(y) e^{\beta(r-|r \omega-y|)} \mathrm{d} y-\int_{I_{1}(r, \omega)} f(y) e^{\beta \omega \cdot y} \mathrm{~d} y\right| \\
\leq & \varepsilon \int_{\mathbf{R}^{N}} f(y) e^{\beta|y|} \mathrm{d} y=C(f) \varepsilon .
\end{aligned}
$$

On the other hand, from $r-|r \omega-y| \leq|y|$, it follows that

$$
\begin{aligned}
& \limsup _{r \rightarrow \infty} \sup _{\omega \in \mathbf{S}^{N-1}} \int_{I_{2}(r, \omega)} f(y) e^{\beta(r-|r \omega-y|)}\left(\frac{1+r}{1+|r \omega-y|}\right)^{\alpha} \mathrm{d} y \\
\leq & \limsup \sup _{r \rightarrow \infty} \int_{\omega \in \mathbf{S}^{N-1}} f(y) e^{\beta|y|}\left(\frac{1+|r \omega-y|+|y|}{1+|r \omega-y|}\right)^{\alpha} \mathrm{d} y \\
\leq & \limsup \sup _{r \rightarrow \infty} \int_{\omega \in \mathbf{S}^{N-1}} \int_{I_{2}(r, \omega)} f(y) e^{\beta|y|}(1+|y|)^{\alpha} \mathrm{d} y=0 .
\end{aligned}
$$

Now from (3.5) through (3.8), we obtain

$$
\limsup _{r \rightarrow \infty} \sup _{\omega \in \mathbf{S}^{N-1}}\left|(1+r)^{\alpha} e^{\beta r} \int_{\mathbf{R}^{N}} g(r \omega-y) f(y) \mathrm{d} y-\gamma \int_{\mathbf{R}^{N}} f(y) e^{\beta \omega \cdot y} \mathrm{~d} y\right| \leq C(f) \varepsilon .
$$

Since $\varepsilon>0$ is arbitrary, Lemma 3.3 holds.

Now we prove Lemma 3.1.

Proof of Lemma 3.1. We first remark that by the strong maximum principle, if $u_{j}(x) \not \equiv 0$, then $u_{j}(x)>$ 0 holds for each $x \in \mathbf{R}^{N}$. We divide our proof into several steps.

Step 1: Proof of (ii).

To this end, it suffices to observe a positive solution of

$$
-\Delta u+(\lambda+V(x)) u=\mu u^{2 p+1} \quad \text { in } \mathbf{R}^{N}, \quad u \in H^{1}\left(\mathbf{R}^{N}\right)
$$

where $\lambda, \mu>0, V(x) \leq 0$ and $V(x) \rightarrow 0$ as $|x| \rightarrow \infty$. We shall prove that for every $\nu \in(0, \lambda)$, there exist $c_{0}>0$, which is independent of $\nu$, and $C_{\nu}>0$ such that

$$
c_{0}(1+|x|)^{-(N-1) / 2} \exp (-\sqrt{\lambda}|x|) \leq u(x) \leq C_{\nu} \exp (-\sqrt{\nu}|x|) \quad \text { for all } x \in \mathbf{R}^{N} .
$$

Let $0<\nu<\lambda$. Since $V(x), u(x) \rightarrow 0$ as $|x| \rightarrow \infty$, we can choose an $R_{\nu}>0$ such that

$$
|x| \geq R_{\nu} \quad \Rightarrow \quad u(x) \leq 1, \quad \lambda+V(x)-\mu u^{2 p}(x) \geq \frac{\lambda+\nu}{2} .
$$

Therefore, $u$ satisfies

$$
-\Delta u+\frac{\lambda+\nu}{2} u \leq 0 \quad \text { in }\left[|x|>R_{\nu}\right]
$$


On the other hand, set

$$
\psi_{\nu}(r):=\exp \left(-\sqrt{\nu}\left(r-R_{\nu}\right)\right)
$$

Noting

$$
\left|\psi_{\nu}^{\prime}(r)\right|=\sqrt{\nu} \psi_{\nu}(r), \quad \psi_{\nu}^{\prime \prime}(r)=\nu \psi_{\nu}(r)
$$

we obtain

$$
\begin{aligned}
-\Delta\left(\psi_{\nu}(|x|)\right)+\frac{\lambda+\nu}{2} \psi_{\nu}(|x|) & =-\psi_{\nu}^{\prime \prime}(|x|)-\frac{N-1}{|x|} \psi_{\nu}^{\prime}(|x|)+\frac{\lambda+\nu}{2} \psi_{\nu}(|x|) \\
& \geq-\frac{N-1}{|x|} \sqrt{\nu} \psi_{\nu}(|x|)+\frac{\lambda-\nu}{2} \psi_{\nu}(|x|) .
\end{aligned}
$$

Thus, enlarging $R_{\nu}$ if necessary, we may assume that

$$
\frac{N-1}{R_{\nu}} \sqrt{\nu} \leq \frac{\lambda-\nu}{2}
$$

From this, it follows that

$$
-\Delta u+\frac{\lambda+\nu}{2} u \leq 0 \leq-\Delta \psi_{\nu}(|x|)+\frac{\lambda+\nu}{2} \psi_{\nu} \quad \text { in }\left[|x|>R_{\nu}\right] .
$$

Noting $u(x) \leq 1=\psi_{\nu}(|x|)$ on $\partial B_{R_{\nu}}(0)$ and $\psi_{\nu}(x), u(x) \rightarrow 0$ as $|x| \rightarrow \infty$, we find that $\psi_{\nu}-u$ does not take a negative minimum in $\left[|x|>R_{\nu}\right]$. Hence, $u(x) \leq \psi_{\nu}(x)$ in $\left[|x|>R_{\nu}\right]$ and this implies

$$
u(x) \leq C_{\nu} \exp (-\sqrt{\nu}|x|) \quad \text { for all } x \in \mathbf{R}^{N} .
$$

Since $\nu \in(0, \lambda)$ is arbitrary, we have the upper estimates in (3.9).

On the other hand, let $G_{\lambda}$ be the Green function for $-\Delta+\lambda$. It is known that

$$
\begin{aligned}
& G_{\lambda} \in C\left(\mathbf{R}^{N} \backslash\{0\}\right) \cap L^{1}\left(\mathbf{R}^{N}\right), \quad G_{\lambda}(x)>0 \quad \text { in } \mathbf{R}^{N} \backslash\{0\}, \\
& \lim _{|x| \rightarrow \infty} G_{\lambda}(x)|x|^{(N-1) / 2} e^{\sqrt{\lambda}|x|}=\gamma_{0} \in(0, \infty) .
\end{aligned}
$$

For instance, see [19]. Since $V(x) \leq 0$ and $u>0$, we have

$$
-\Delta G_{\lambda}+\lambda G_{\lambda}=0 \leq-V(x) u+\mu u^{2 p+1}=-\Delta u+\lambda u \quad \text { in }[|x|>1] .
$$

Noting $G_{\lambda}(x), u(x) \rightarrow 0$ as $|x| \rightarrow \infty$ and choosing $c_{1}>0$ so that $c_{1} G_{\lambda}(x) \leq u(x)$ for any $|x|=1$, we see that $u(x)-c_{1} G_{\lambda}(x)$ does not have negative minimum on $[|x|>1]$. Thus $u-c_{1} G_{\lambda} \geq 0$ in $[|x| \geq 1]$ and it follows from (3.10) that

$$
c_{0}(1+|x|)^{-(N-1) / 2} \exp (-\sqrt{\lambda}|x|) \leq u(x) \quad \text { for all } x \in \mathbf{R}^{N} .
$$

Hence, (3.9) and Step 1 hold.

Next, we treat (i) and (iii). Note that when $u_{2} \equiv 0$, (i) is derived from (3.9). Since the condition $u_{1}, u_{2}>0$ is assumed in (iii), in what follows, we may suppose $u_{1}, u_{2}>0$ without loss of generality.

Step 2: Proof of (i).

Set $u(x):=u_{1}(x)+u_{2}(x)$. It follows from (3.1) that

$$
\begin{aligned}
& -\Delta u+\lambda_{0,1} u+\left\{V_{1}(x) u_{1}+\left(V_{2}(x)+\lambda_{0,2}-\lambda_{0,1}\right) u_{2}\right\} \\
= & \mu_{1} u_{1}^{2 p_{1}+1}+\mu_{2} u_{2}^{2 p_{2}+1}+\beta\left(u_{1}^{p_{3}} u_{2}^{p_{3}+1}+u_{1}^{p_{3}+1} u_{2}^{p_{3}}\right) .
\end{aligned}
$$

Recalling $V_{j}(x) \leq 0, u_{j}(x)>0$ and (3.2), we obtain

$$
-\Delta u+\lambda_{0,1} u+2 V(x) u \leq \mu_{1} u^{2 p_{1}+1}+\mu_{2} u^{2 p_{2}+1}+2 \beta u^{2 p_{3}+1} \quad \text { in } \mathbf{R}^{N}
$$

where $V(x):=V_{1}(x)+V_{2}(x)$. Hence, for $u$, we can apply the above argument and show that for each $\lambda \in\left(0, \lambda_{0,1}\right)$ there exists a $C_{\lambda}>0$ so that

$$
u_{1}(x)+u_{2}(x)=u(x) \leq C_{\lambda} \exp (-\sqrt{\lambda}|x|) \quad \text { for all } x \in \mathbf{R}^{N} .
$$


On the other hand, since

$$
-\Delta u_{1}+\lambda_{0,1} u_{1}=-V(x) u_{1}+\mu_{1} u_{1}^{2 p_{1}+1}+\beta u_{1}^{p_{3}} u_{2}^{p_{3}+1} \geq 0=-\Delta G_{\lambda_{0,1}}+\lambda_{0,1} G_{\lambda_{0,1}} \quad \text { in }[|x|>1],
$$

as in the above, we may find a $c_{0}>0$ such that

$$
c_{0}(1+|x|)^{-(N-1) / 2} \exp \left(-\sqrt{\lambda_{0,1}}|x|\right) \leq u_{1}(x) \quad \text { for each } x \in \mathbf{R}^{N} .
$$

Hence, (i) holds.

Step 3: Proof of (iii) when either $p_{3} \geq 1$ or else $0<p_{3}<1$ and $\lambda_{0,3}=\left(1+p_{3}\right)^{2} \lambda_{0,1} /\left(1-p_{3}\right)^{2}>\lambda_{0,2}$.

When $1 \leq p_{3}$, notice that $u_{2}$ satisfies

$$
-\Delta u_{2}+\left(\lambda_{0,2}+V_{2}(x)-\mu_{2} u_{2}^{2 p_{2}}-\beta u_{1}^{p_{3}+1} u_{2}^{p_{3}-1}\right) u_{2}=0 \quad \text { in } \mathbf{R}^{N} .
$$

Since $V_{2}(x), u_{1}(x), u_{2}(x) \rightarrow 0$ as $|x| \rightarrow \infty$, we may use the argument in Step 1 to prove that for each $\lambda \in\left(0, \lambda_{0,2}\right)$ we find a $C_{\lambda}>0$ with

$$
u_{2}(x) \leq C_{\lambda} \exp (-\sqrt{\lambda}|x|) \quad \text { for all } x \in \mathbf{R}^{N} .
$$

On the other hand, from

$$
-\Delta u_{2}+\lambda_{0,2} u_{2}=-V_{2}(x) u_{2}+\mu_{2} u_{2}^{2 p_{2}+1}+\beta u_{1}^{p_{3}+1} u_{2}^{p_{3}} \geq 0=-\Delta G_{\lambda_{0,2}}+\lambda_{0,2} G_{\lambda_{0,2}} \quad \text { in }[|x|>1],
$$

it follows that for some $c_{0}>0$,

$$
c_{0}(1+|x|)^{-(N-1) / 2} \exp \left(-\sqrt{\lambda_{0,2}}|x|\right) \leq u_{2}(x) \quad \text { for each } x \in \mathbf{R}^{N} .
$$

Hence, when $p_{3} \geq 1$, (iii) holds.

Next, suppose $0<p_{3}<1$ and $\lambda_{0,3}>\lambda_{0,2}$. Since the lower estimate for $u_{2}$ can be shown as in the above, we only prove the upper estimate. Set

$$
f_{0}(x):=\mu_{2} u_{2}^{2 p_{2}+1}(x)+\beta u_{1}^{p_{3}+1}(x) u_{2}^{p_{3}}(x) .
$$

By (3.11), we may find $\xi_{1}>\lambda_{0,1}$ such that

$$
f_{0}(x) \leq C_{0} \exp \left(-\sqrt{\xi_{1}}|x|\right) \quad \text { for all } x \in \mathbf{R}^{N} .
$$

Let $\nu>0$ and set $\varphi_{\nu}(x):=C \exp (-\sqrt{\nu}|x|)$. Then we have

$$
-\Delta \varphi_{\nu}+\left(\lambda_{0,2}+V_{2}(x)\right) \varphi_{\nu} \geq\left(\lambda_{0,2}+V_{2}(x)-\nu-\frac{N-1}{|x|} \sqrt{\nu}\right) \varphi_{\nu} .
$$

Therefore, if $\nu<\min \left\{\lambda_{0,2}, \xi_{1}\right\}$, then we may find an $R_{\nu}>0$ such that

$$
-\Delta \varphi_{\nu}+\left(\lambda_{0,2}+V_{2}(x)\right) \varphi_{\nu} \geq f_{0}(x)=-\Delta u_{2}+\left(\lambda_{0,2}+V_{2}(x)\right) u_{2} \quad \text { in }\left[|x|>R_{\nu}\right] .
$$

Hence, for all $\nu \in\left(0, \min \left\{\lambda_{0,2}, \xi_{1}\right\}\right)$,

$$
u_{2}(x) \leq C_{\nu} \exp (-\sqrt{\nu}|x|) \quad \text { for every } x \in \mathbf{R}^{N} .
$$

If $\xi_{1} \geq \lambda_{0,2}$, then (3.14) is the desired estimate and we complete the proof. When $\xi_{1}<\lambda_{0,2}$, for any $\varepsilon \in\left(0, \lambda_{0,1} / 2\right)$, set

$$
\sqrt{\xi_{2, \varepsilon}}:=\min \left\{\left(2 p_{2}+1\right) \sqrt{\xi_{1}-\varepsilon},\left(p_{3}+1\right) \sqrt{\lambda_{0,1}-\varepsilon}+p_{3} \sqrt{\xi_{1}-\varepsilon}\right\} .
$$

By the definition of $f_{0}$ and (3.14), we have

$$
f_{0}(x) \leq C_{\varepsilon} \exp \left(-\sqrt{\xi_{2, \varepsilon}}|x|\right) \quad \text { for all } x \in \mathbf{R}^{N} .
$$

Repeating the argument above, for each $0<\varepsilon<\lambda_{0,1} / 2$ and $0<\nu<\min \left\{\lambda_{0,2}, \xi_{2, \varepsilon}\right\}$, we obtain (3.13) and

$$
u_{2}(x) \leq C_{\nu} \exp (-\sqrt{\nu}|x|) \quad \text { for every } x \in \mathbf{R}^{N} .
$$


When $\xi_{2, \varepsilon} \geq \lambda_{0,2}$ for some $\varepsilon \in\left(0, \lambda_{0,1} / 2\right)$, we complete the proof. If $\xi_{2, \varepsilon}<\lambda_{0,2}$ for any $\varepsilon \in\left(0, \lambda_{0,1} / 2\right)$, define

Then from

$$
\sqrt{\xi_{3, \varepsilon}}:=\min \left\{\left(2 p_{2}+1\right) \sqrt{\xi_{2, \varepsilon}},\left(p_{3}+1\right) \sqrt{\lambda_{0,1}-\varepsilon}+p_{3} \sqrt{\xi_{2, \varepsilon}}\right\} .
$$

$$
f_{0}(x) \leq C_{\varepsilon} \exp \left(-\sqrt{\xi_{3, \varepsilon}}|x|\right) \quad \text { for all } x \in \mathbf{R}^{N},
$$

it follows that for each $0<\varepsilon<\lambda_{0,1} / 2$ and $0<\nu<\min \left\{\lambda_{0,2}, \xi_{3, \varepsilon}\right\}$,

$$
u_{2}(x) \leq C_{\nu} \exp (-\sqrt{\nu}|x|) \quad \text { for every } x \in \mathbf{R}^{N} .
$$

By repeating the same procedure, if $\xi_{n, \varepsilon}<\lambda_{0,2}$ holds for all $\varepsilon \in\left(0, \lambda_{0,1} / 2\right)$, then define

$$
\sqrt{\xi_{n+1, \varepsilon}}:=\min \left\{\left(2 p_{2}+1\right) \sqrt{\xi_{n, \varepsilon}},\left(p_{3}+1\right) \sqrt{\lambda_{0,1}-\varepsilon}+p_{3} \sqrt{\xi_{n, \varepsilon}}\right\} .
$$

To prove (iii), it suffices to show that there are $\varepsilon_{0} \in\left(0, \lambda_{0,1} / 2\right)$ and $n_{0}$ such that $\xi_{n_{0}, \varepsilon_{0}} \geq \lambda_{0,2}$. If this is not true, then

$$
\xi_{n, \varepsilon}<\lambda_{0,2} \quad \text { for every }(\varepsilon, n) \in\left(0, \frac{\lambda_{0,1}}{2}\right) \times \mathbf{N} .
$$

By the assumption $\left(1+p_{3}\right)^{2} \lambda_{0,1} /\left(1-p_{3}\right)^{2}=\lambda_{0,3}>\lambda_{0,2}$, we may select an $\varepsilon_{0} \in\left(0, \lambda_{0,1} / 2\right)$ so that

$$
\frac{\left(1+p_{3}\right)^{2}}{\left(1-p_{3}\right)^{2}}\left(\lambda_{0,1}-\varepsilon_{0}\right)>\lambda_{0,2}
$$

Since $0<p_{3}<1, \sqrt{s}<\left(2 p_{2}+1\right) \sqrt{s}$ for $s>0$ and

$$
\sqrt{s}<\left(p_{3}+1\right) \sqrt{\lambda_{0,1}-\varepsilon_{0}}+p_{3} \sqrt{s} \Leftrightarrow s<\frac{\left(1+p_{3}\right)^{2}}{\left(1-p_{3}\right)^{2}}\left(\lambda_{0,1}-\varepsilon_{0}\right),
$$

it follows from (3.15)-(3.18) with $s=\xi_{n, \varepsilon}$ that $\xi_{n, \varepsilon_{0}}<\xi_{n+1, \varepsilon_{0}}<\lambda_{0,2}$.

Let $\xi_{\infty, \varepsilon_{0}}:=\lim _{n \rightarrow \infty} \xi_{n, \varepsilon_{0}} \leq \lambda_{0,2}$. Then,

$$
\sqrt{\xi_{\infty, \varepsilon_{0}}} \leq\left(p_{3}+1\right) \sqrt{\lambda_{0,1}-\varepsilon_{0}}+p_{3} \sqrt{\xi_{\infty, \varepsilon_{0}}} .
$$

If $\sqrt{\xi_{\infty, \varepsilon_{0}}}<\left(p_{3}+1\right) \sqrt{\lambda_{0,1}-\varepsilon_{0}}+p_{3} \sqrt{\xi_{\infty, \varepsilon_{0}}}$, then for sufficiently large $n$, it follows that

$$
\sqrt{\xi_{\infty, \varepsilon_{0}}}<\left(p_{3}+1\right) \sqrt{\lambda_{0,1}-\varepsilon_{0}}+p_{3} \sqrt{\xi_{n, \varepsilon_{0}}}, \quad \sqrt{\xi_{\infty, \varepsilon_{0}}}<\left(2 p_{1}+1\right) \sqrt{\xi_{n, \varepsilon_{0}}} .
$$

From the definition of $\xi_{n+1, \varepsilon_{0}}$, we have a contradiction: $\sqrt{\xi_{\infty, \varepsilon_{0}}}<\sqrt{\xi_{n+1, \varepsilon_{0}}}$.

Now, we get

$$
\sqrt{\xi_{\infty, \varepsilon_{0}}}=\left(p_{3}+1\right) \sqrt{\lambda_{0,1}-\varepsilon_{0}}+p_{3} \sqrt{\xi_{\infty, \varepsilon_{0}}} .
$$

However, by (3.17) and $\xi_{\infty, \varepsilon_{0}} \leq \lambda_{0,2}$, we have a contradiction: $\xi_{\infty, \varepsilon_{0}}=\left(1+p_{3}\right)^{2}\left(\lambda_{0,1}-\varepsilon_{0}\right) /\left(1-p_{3}\right)^{2}>$ $\lambda_{0,2}$. Therefore Step 3 holds.

Step 4: Proof of (iii) when $0<p_{3}<1$ and $\left(1+p_{3}\right)^{2} \lambda_{0,1} /\left(1-p_{3}\right)^{2}=\lambda_{0,3} \leq \lambda_{0,2}$.

We first consider the upper estimate for $u_{2}$. Remark that $\lambda_{0,1}<\lambda_{0,2}$ holds in this case. Hence, at the estimate (3.12), we may suppose

$$
\lambda_{0,1}<\xi_{1}<\frac{\left(1+p_{3}\right)^{2}}{\left(1-p_{3}\right)^{2}} \lambda_{0,1} \leq \lambda_{0,2}
$$

and fix an $\varepsilon_{1} \in\left(0, \lambda_{0,1} / 2\right)$ so that

$$
\lambda_{0,1}<\xi_{1}<\frac{\left(1+p_{3}\right)^{2}}{\left(1-p_{3}\right)^{2}}\left(\lambda_{0,1}-\varepsilon_{1}\right) .
$$

We argue as in Step 3 and define $\left(\xi_{n, \varepsilon}\right)$ by $(3.15)$ with $\xi_{1, \varepsilon}:=\xi_{1}-\varepsilon$ for $\varepsilon \in\left(0, \varepsilon_{1}\right)$, that is,

$$
\sqrt{\xi_{n+1, \varepsilon}}:=\min \left\{\left(2 p_{2}+1\right) \sqrt{\xi_{n, \varepsilon}},\left(p_{3}+1\right) \sqrt{\lambda_{0,1}-\varepsilon}+p_{3} \sqrt{\xi_{n, \varepsilon}}\right\} \quad \text { for } n \geq 1 \text {. }
$$


Recalling (3.18) and (3.19), inductively, we can show that for each $n \geq 1$,

$$
\begin{aligned}
\sqrt{\xi_{n+1, \varepsilon}} \leq\left(p_{3}+1\right) \sqrt{\lambda_{0,1}-\varepsilon}+p_{3} \sqrt{\xi_{n, \varepsilon}} & \leq\left(p_{3}+1\right) \sqrt{\lambda_{0,1}-\varepsilon}+p_{3} \sqrt{\xi_{\infty, \varepsilon}}=\sqrt{\xi_{\infty, \varepsilon}}, \\
\xi_{n, \varepsilon} & \leq \xi_{n+1, \varepsilon}
\end{aligned}
$$

where

$$
\xi_{\infty, \varepsilon}:=\frac{\left(1+p_{3}\right)^{2}}{\left(1-p_{3}\right)^{2}}\left(\lambda_{0,1}-\varepsilon\right) .
$$

As before, it is not difficult to check $\xi_{n, \varepsilon} \rightarrow \xi_{\infty, \varepsilon}$. Furthermore, $\xi_{\infty, \varepsilon} \rightarrow \lambda_{0,3}$ as $\varepsilon \rightarrow 0$. Since for each $0<\varepsilon<\varepsilon_{1}$ and $0<\nu<\min \left\{\xi_{n+1, \varepsilon}, \lambda_{0,2}\right\}$, one finds a $C_{\nu}>0$ so that

$$
u_{2}(x) \leq C_{\nu} \exp (-\sqrt{\nu}|x|) \quad \text { for each } x \in \mathbf{R}^{N},
$$

we may infer that for each $0<\nu<\lambda_{0,3}$ there exists a $C_{\nu}>0$ with

$$
u_{2}(x) \leq C_{\nu} \exp (-\sqrt{\nu}|x|) \quad \text { for all } x \in \mathbf{R}^{N} .
$$

Next, we show the lower estimate for $u_{2}$. First, as in Step 3, we have

$$
c_{0}(1+|x|)^{-(N-1) / 2} \exp \left(-\sqrt{\lambda_{0,2}}|x|\right) \leq u_{2}(x) .
$$

Thus, if

$$
\lambda_{0,3}=\frac{\left(1+p_{3}\right)^{2}}{\left(1-p_{3}\right)^{2}} \lambda_{0,1}=\lambda_{0,2},
$$

then our assertion clearly holds. Therefore, we may suppose $\lambda_{0,3}<\lambda_{0,2}$. Set

$$
\sqrt{\zeta_{1}}:=\left(p_{3}+1\right) \sqrt{\lambda_{0,1}}+p_{3} \sqrt{\lambda_{0,2}} .
$$

By (3.18) with $\varepsilon_{0}=0$, we have

$$
\sqrt{\lambda_{0,3}}=\left(p_{3}+1\right) \sqrt{\lambda_{0,1}}+p_{3} \sqrt{\lambda_{0,3}}<\left(p_{3}+1\right) \sqrt{\lambda_{0,1}}+p_{3} \sqrt{\lambda_{0,2}}=\sqrt{\zeta_{1}}<\sqrt{\lambda_{0,2}} .
$$

Then by (i) and (3.20), for some $\alpha_{1}>0$ and $\tilde{c}_{1}>0$,

$$
f_{0}(x) \geq \beta u_{1}^{p_{3}+1}(x) u_{2}^{p_{3}}(x) \geq \tilde{c}_{1}(1+|x|)^{-\alpha_{1}} \exp \left(-\sqrt{\zeta_{1}}|x|\right) .
$$

Noting

$$
-\Delta u_{2}+\lambda_{0,2} u_{2}=-V_{2}(x) u_{2}+f_{0}(x) \quad \text { in } \mathbf{R}^{N}
$$

and $V_{2} \leq 0$ and recalling the Green function $G_{\lambda_{0,2}}$, we have

$$
\begin{aligned}
u_{2}(x) & =\int_{\mathbf{R}^{N}} G_{\lambda_{0,2}}(x-y)\left(-V_{2}(y) u_{2}(y)+f_{0}(y)\right) \mathrm{d} y \\
& \geq \tilde{c}_{1} \int_{\mathbf{R}^{N}} G_{\lambda_{0,2}}(x-y)(1+|y|)^{-\alpha_{1}} \exp \left(-\sqrt{\zeta_{1}}|y|\right) \mathrm{d} y .
\end{aligned}
$$

By (3.10), (3.21) and applying Lemma 3.3, one sees that

$$
\begin{aligned}
& (1+r)^{\alpha_{1}} \exp \left(\sqrt{\zeta_{1}} r\right) \int_{\mathbf{R}^{N}} G_{\lambda_{0,2}}(r \omega-y)(1+|y|)^{-\alpha_{1}} \exp \left(-\sqrt{\zeta_{1}}|y|\right) \mathrm{d} y \\
\rightarrow & \int_{\mathbf{R}^{N}} G_{\lambda_{0,2}}(y) e^{\sqrt{\zeta_{1}} \omega \cdot y} \mathrm{~d} y>0 \quad \text { unifromly with respect to } \omega \in \mathbf{S}^{N-1} .
\end{aligned}
$$

Hence, for some $c_{1}>0$,

$$
u_{2}(x) \geq c_{1}(1+|x|)^{-\alpha_{1}} \exp \left(-\sqrt{\zeta_{1}}|x|\right) \quad \text { for each } x \in \mathbf{R}^{N} .
$$

Next, set

$$
\sqrt{\zeta_{2}}:=\left(p_{3}+1\right) \sqrt{\lambda_{0,1}}+p_{3} \sqrt{\zeta_{1}} .
$$

Then arguing as in the above, we have

$$
\lambda_{0,3}<\zeta_{2}<\zeta_{1}
$$


and for some $\tilde{c}_{2}>0$ and $\alpha_{2}>0$,

$$
f_{0}(x) \geq \beta u_{1}^{p_{3}+1}(x) u_{2}^{p_{3}}(x) \geq \tilde{c}_{2}(1+|x|)^{-\alpha_{2}} \exp \left(-\sqrt{\zeta_{2}}|x|\right) .
$$

From this and Lemma 3.3, for some $c_{2}>0$, it follows that

Inductively, we define

$$
u_{2}(x) \geq c_{2}(1+|x|)^{-\alpha_{2}} \exp \left(-\sqrt{\zeta_{2}}|x|\right) .
$$

$$
\zeta_{n+1}:=\left(p_{3}+1\right) \sqrt{\lambda_{0,1}}+p_{3} \sqrt{\zeta_{n}}
$$

Then we can check that

$$
\begin{aligned}
& \lambda_{0,3}<\zeta_{n+1}<\zeta_{n}, \quad f_{0}(x) \geq \tilde{c}_{n+1}(1+|x|)^{-\alpha_{n+1}} \exp \left(-\sqrt{\zeta_{n+1}}|x|\right), \\
& u_{2}(x) \geq c_{n+1}(1+|x|)^{-\alpha_{n+1}} \exp \left(-\sqrt{\zeta_{n+1}}|x|\right) .
\end{aligned}
$$

Finally, since $\lambda_{0,3}<\zeta_{n+1}<\zeta_{n}$, it is easily seen that $\zeta_{n} \rightarrow \lambda_{0,3}$. Hence, the desired lower estimate holds and we complete the proof.

3.2. Inequality for the interaction estimate. In this subsection, we prepare some inequalities for the interaction estimate:

Lemma 3.4. Let $p>0$.

(i) There exists $a C_{p}>0$ such that for any $a, b \geq 0$,

$$
(a+b)^{2 p+2} \geq a^{2 p+2}+b^{2 p+2}+(2 p+2)\left(a^{2 p+1} b+a b^{2 p+1}\right)-C_{p} a^{p+1} b^{p+1} .
$$

(ii) Let $\eta \in(0, p)$. Then there exists a $C_{p, \eta}>0$ such that for any $a_{i}, b_{i} \geq 0$,

$$
\begin{aligned}
\left(a_{1}+b_{1}\right)^{p+1}\left(a_{2}+b_{2}\right)^{p+1} \geq & a_{1}^{p+1} a_{2}^{p+1}+b_{1}^{p+1} b_{2}^{p+1}+(p+1)\left(a_{1}^{p} a_{2}^{p+1} b_{1}+a_{1}^{p+1} a_{2}^{p} b_{2}+a_{2} b_{1}^{p+1} b_{2}^{p}\right) \\
& -C_{p, \eta}\left(a_{1}^{p-\eta} a_{2}^{p+1} b_{1}^{1+\eta}+a_{1}^{1+\eta} b_{2}^{p+1} b_{1}^{p-\eta}\right) .
\end{aligned}
$$

Proof. The assertion (i) is proved in [3, Lemma 2.1]. For the assertion (ii), it is enough to consider $b_{1}, b_{2}>0$. Dividing by $b_{1}^{p+1} b_{2}^{p+1}$ and setting $x=a_{1} / b_{1}$ and $y=a_{2} / b_{2}$, we shall show that for each $x, y \geq 0$

$$
\begin{aligned}
0 \leq f(x, y):= & (x+1)^{p+1}(y+1)^{p+1}-x^{p+1} y^{p+1}-1 \\
& -(p+1)\left(x^{p} y^{p+1}+x^{p+1} y^{p}+y\right)+C_{p, \eta}\left(x^{p-\eta} y^{p+1}+x^{1+\eta}\right) .
\end{aligned}
$$

In what follows, we may assume $C_{p, \eta} \geq 1$ and divide our proof into three steps.

Step 1: There exists an $x_{1}>0$ such that $f(x, y) \geq 0$ for all $(x, y) \in\left[0, x_{1}\right] \times[0, \infty)$.

By simple computations, we obtain

$$
\begin{aligned}
\frac{\partial f}{\partial x}=( & +1)(x+1)^{p}(y+1)^{p+1}-(p+1) x^{p} y^{p+1}-(p+1)\left[p x^{p-1} y^{p+1}+(p+1) x^{p} y^{p}\right] \\
& +C_{p, \eta}\left[(p-\eta) x^{p-\eta-1} y^{p+1}+(1+\eta) x^{\eta}\right] \\
= & (p+1)\left[(x+1)^{p}(y+1)^{p+1}-x^{p} y^{p+1}-(p+1) x^{p} y^{p}\right] \\
& +x^{p-\eta-1} y^{p+1}\left[(p-\eta) C_{p, \eta}-p(p+1) x^{\eta}\right]+C_{p, \eta}(1+\eta) x^{\eta}
\end{aligned}
$$

Let

$$
x^{p} \leq \frac{1}{2(p+1)}<\frac{1}{2} .
$$

From $\max \left\{y^{p}, y^{p+1}\right\} \leq(1+y)^{p+1}$ for every $y \geq 0$, it follows that

$$
(x+1)^{p}(y+1)^{p+1}-x^{p} y^{p+1}-(p+1) x^{p} y^{p} \geq(y+1)^{p+1}-\frac{1}{2} y^{p+1}-\frac{1}{2} y^{p} \geq 0 \quad \text { for any } y \geq 0 .
$$

On the other hand, if

$$
x^{\eta} \leq \frac{p-\eta}{p(p+1)}
$$


then from $C_{p, \eta} \geq 1$ we see that

$$
(p-\eta) C_{p, \eta}-p(p+1) x^{\eta} \geq 0
$$

Thus choosing

$$
x_{1}:=\min \left\{\left[\frac{1}{2(p+1)}\right]^{1 / p},\left[\frac{p-\eta}{p(p+1)}\right]^{1 / \eta}\right\}>0,
$$

we obtain

$$
\frac{\partial f}{\partial x}(x, y) \geq 0 \text { for each }(x, y) \in\left[0, x_{1}\right] \times[0, \infty) .
$$

By $f(0, y)=(y+1)^{p+1}-1-(p+1) y \geq 0$ for all $y \geq 0$, Step 1 holds.

Step 2: There exists a $y_{1}>0$ such that $f(x, y) \geq 0$ for all $(x, y) \in\left[x_{1}, \infty\right) \times\left[0, y_{1}\right]$.

Let $x \geq x_{1}$. Since $C_{p, \eta} \geq 1$ and $(y+1)^{p+1} \geq 1+(p+1) y$ holds for each $y \geq 0$, we have

$$
\begin{aligned}
f(x, y) \geq & (x+1)^{p+1}[1+(p+1) y]-x^{p+1} y^{p+1}-1-(p+1)\left(x^{p} y^{p+1}+x^{p+1} y^{p}+y\right)+x_{1}^{1+\eta} \\
= & {\left[(x+1)^{p+1}-1-(p+1) x^{p+1} y^{p}\right]+\left[x_{1}^{1+\eta}-(p+1) y\right] } \\
& +y\left[(p+1)(x+1)^{p+1}-x^{p+1} y^{p}-(p+1) x^{p} y^{p}\right] .
\end{aligned}
$$

Noting $x \geq x_{1}$, we may find a $c_{x_{1}} \in(0,1)$ so that

$$
(x+1)^{p+1}-1 \geq c_{x_{1}}(x+1)^{p+1} \text { for every } x \in\left[x_{1}, \infty\right) .
$$

If

$$
y^{p} \leq \frac{c_{x_{1}}}{p+1}
$$

then we obtain

$$
(x+1)^{p+1}-1-(p+1) x^{p+1} y^{p} \geq c_{x_{1}}(x+1)^{p+1}-c_{x_{1}} x^{p+1}>0 \text { for every } x \in\left[x_{1}, \infty\right) .
$$

On the other hand, if

$$
y^{p} \leq \frac{p+1}{p+2}
$$

then for every $x \in\left[x_{1}, \infty\right)$,

$$
(p+1)(x+1)^{p+1}-x^{p+1} y^{p}-(p+1) x^{p} y^{p} \geq(x+1)^{p+1}\left[p+1-y^{p}-(p+1) y^{p}\right] \geq 0 .
$$

Hence, setting

$$
y_{1}:=\min \left\{\left(\frac{c_{x_{1}}}{p+1}\right)^{1 / p},\left(\frac{p+1}{p+2}\right)^{1 / p}, \frac{x_{1}^{1+\eta}}{p+1}\right\}>0
$$

we see that

$$
f(x, y) \geq 0 \quad \text { for each }(x, y) \in\left[x_{1}, \infty\right) \times\left[0, y_{1}\right] .
$$

Step 3: $f(x, y) \geq 0$ for all $(x, y) \in\left[x_{1}, \infty\right) \times\left[y_{1}, \infty\right)$.

Let $x \geq x_{1}$ and $y \geq y_{1}$. Note that

$$
\begin{aligned}
& x^{-p-1} f(x, y) \\
= & \left(1+\frac{1}{x}\right)^{p+1}(y+1)^{p+1}-y^{p+1}-\frac{1}{x^{p+1}}-(p+1)\left(\frac{y^{p+1}}{x}+y^{p}+\frac{y}{x^{p+1}}\right)+C_{p, \eta}\left(\frac{y^{p+1}}{x^{1+\eta}}+x^{\eta-p}\right) .
\end{aligned}
$$


Then we observe that for every $(x, y) \in\left[x_{1}, \infty\right) \times\left[y_{1}, \infty\right)$

$$
\begin{aligned}
& \left(1+\frac{1}{x}\right)^{p+1}(y+1)^{p+1}-y^{p+1}-(p+1)\left(\frac{y^{p+1}}{x}+y^{p}\right) \\
\geq & \left(1+\frac{p+1}{x}\right)(y+1)^{p+1}-y^{p+1}-(p+1) \frac{y^{p+1}}{x}-(p+1) y^{p} \\
= & {\left[(y+1)^{p+1}-y^{p+1}-(p+1) y^{p}\right]+\frac{p+1}{x}\left[(y+1)^{p+1}-y^{p+1}\right] } \\
= & y^{p+1}\left[\left(1+\frac{1}{y}\right)^{p+1}-1-\frac{p+1}{y}\right]+\frac{p+1}{x}\left[(y+1)^{p+1}-y^{p+1}\right] \\
\geq & 0 .
\end{aligned}
$$

On the other hand, choosing

$$
C_{p, \eta} \geq \max \left\{1, \frac{2}{x_{1}^{p-\eta} y_{1}^{p+1}}, \frac{2(p+1)}{x_{1}^{p-\eta} y_{1}^{p}}\right\},
$$

it follows from $0<\eta<p$ that for each $(x, y) \in\left[x_{1}, \infty\right) \times\left[y_{1}, \infty\right)$,

$$
\begin{aligned}
-\frac{1}{x^{p+1}}-\frac{(p+1) y}{x^{p+1}}+C_{p, \eta} \frac{y^{p+1}}{x^{1+\eta}} & =\frac{1}{x^{1+\eta}}\left[\left(\frac{C_{p, \eta} y^{p+1}}{2}-\frac{1}{x^{p-\eta}}\right)+y\left(\frac{C_{p, \eta} y^{p}}{2}-\frac{p+1}{x^{p-\eta}}\right)\right] \\
& \geq \frac{1}{x^{1+\eta}}\left[\left(\frac{C_{p, \eta} y_{1}^{p+1}}{2}-\frac{1}{x_{1}^{p-\eta}}\right)+y\left(\frac{C_{p, \eta} y_{1}^{p}}{2}-\frac{p+1}{x_{1}^{p-\eta}}\right)\right] \geq 0 .
\end{aligned}
$$

Hence, $f(x, y) \geq 0$ for each $(x, y) \in\left[x_{1}, \infty\right) \times\left[y_{1}, \infty\right)$ and Step 3 holds.

From Step 1 through Step 3, we complete the proof.

3.3. Proof of Theorem 1.1. In this subsection, we shall prove Theorem 1.1. Let us take any minimizing sequence $\left(u_{n}\right) \subset M(\alpha)$ for $e(\alpha)$. By Lemmas 2.2 and 2.3, without loss of generality, we may further suppose that

$$
E\left(u_{n}\right) \rightarrow e(\alpha), \quad\left\|E^{\prime}\left(u_{n}\right)+\lambda_{n, 1} Q_{1}^{\prime}\left(u_{n}\right)+\lambda_{n, 2} Q_{2}^{\prime}\left(u_{n}\right)\right\|_{H^{*}} \rightarrow 0, \quad\left\|\left(u_{n, i}\right)_{-}\right\|_{L^{2}} \rightarrow 0 .
$$

In addition, since $\left(u_{n}\right)$ and $\left(\lambda_{n, i}\right)$ are bounded, one may assume that $u_{n} \rightarrow u_{0}$ weakly in $H$ and $\lambda_{n, i} \rightarrow \lambda_{0, i}$. Remark that

$$
\gamma_{u_{0}, i}:=\left\|u_{0, i}\right\|_{L^{2}}^{2}=2 Q_{i}\left(u_{0}\right) \leq \alpha_{i} .
$$

Notice that if $\gamma_{u_{0}}=\left(\gamma_{u_{0}, 1}, \gamma_{u_{0}, 2}\right)=\left(\alpha_{1}, \alpha_{2}\right)$, then $\left\|u_{n, i}-u_{0, i}\right\|_{L^{2}}^{2} \rightarrow 0$ with $i=1,2$. Moreover, from (3.22), $e(\alpha) \leq E\left(u_{0}\right)$ and the weak lower semicontinuity, it follows that $\left\|\nabla u_{n, i}\right\|_{L^{2}} \rightarrow\left\|\nabla u_{0, i}\right\|_{L^{2}}$ and $\left\|u_{n}-u_{0}\right\|_{H} \rightarrow 0$.

Now, our aim is to prove $\gamma_{u_{0}}=\left(\alpha_{1}, \alpha_{2}\right)$. We argue by contradiction and suppose that

$$
\gamma_{u_{0}}:=\left(\gamma_{u_{0}, 1}, \gamma_{u_{0}, 2}\right) \neq\left(\alpha_{1}, \alpha_{2}\right) \text {. }
$$

We first prove

Lemma 3.5. Let $Q^{N}:=[0,1]^{N}$. Then $\liminf _{n \rightarrow \infty} \sup _{z \in \mathbf{Z}^{N}}\left\|u_{n}-u_{0}\right\|_{L^{2}\left(Q^{N}+z\right)}>0$.

Proof. Let us suppose $\sup _{z \in \mathbf{Z}^{N}}\left\|u_{n}-u_{0}\right\|_{L^{2}\left(Q^{N}+z\right)} \rightarrow 0$. Then we infer that $u_{n} \rightarrow u_{0}$ strongly in $L^{q}\left(\mathbf{R}^{N}\right)$ for every $2<q<2^{*}$ (See [43], for instance). Therefore, we obtain

$$
e\left(\gamma_{u_{0}}\right) \leq E\left(u_{0}\right) \leq \lim _{n \rightarrow \infty} E\left(u_{n}\right)=e(\alpha) .
$$

On the other hand, by Lemma 2.1(iii), it follows that

$$
e(\alpha) \leq e\left(\gamma_{u_{0}}\right)+e_{\infty}\left(\alpha-\gamma_{u_{0}}\right) .
$$

Since $\gamma_{u_{0}} \neq \alpha$, we have $e_{\infty}\left(\alpha-\gamma_{u_{0}}\right)<0$, which contradicts (3.23). 
Thanks to Lemma 3.5, we may find $\left(y_{n}\right) \subset \mathbf{R}^{N}$ such that $\left\|u_{n}\right\|_{L^{2}\left(Q^{N}+y_{n}\right)} \rightarrow c_{0}>0$ and $\left|y_{n}\right| \rightarrow \infty$. Let

$$
u_{n}\left(\cdot+y_{n}\right) \rightarrow w_{0}=\left(w_{0,1}, w_{0,2}\right) \quad \text { weakly in } H
$$

Remark that

$$
w_{0} \not \equiv(0,0) .
$$

Moreover, it follows from $\left|y_{n}\right| \rightarrow \infty$ as $n \rightarrow \infty$ that for $i=1,2$,

$$
\begin{aligned}
& \left\|u_{n, i}-u_{0, i}-w_{0, i}\left(\cdot-y_{n}\right)\right\|_{L^{2}}^{2} \\
= & \left\|u_{n, i}\right\|_{L^{2}}^{2}+\left\|u_{0, i}\right\|_{L^{2}}^{2}+\left\|w_{0, i}\right\|_{L^{2}}^{2}-2\left\langle u_{n, i}, u_{0, i}\right\rangle_{L^{2}}-2\left\langle u_{n, i}\left(\cdot+y_{n}\right), w_{0, i}\right\rangle_{L^{2}}+o(1) \\
= & \left\|u_{n, i}\right\|_{L^{2}}^{2}-\left\|u_{0, i}\right\|_{L^{2}}^{2}-\left\|w_{0, i}\right\|_{L^{2}}^{2}+o(1) .
\end{aligned}
$$

In particular, we have

$$
\gamma_{w_{0}, i}:=\left\|w_{0, i}\right\|_{L^{2}}^{2} \leq \liminf _{n \rightarrow \infty}\left(\left\|u_{n, i}\right\|_{L^{2}}^{2}-\left\|u_{0, i}\right\|_{L^{2}}^{2}\right)=\alpha_{i}-\gamma_{u_{0}, i} .
$$

Next, we show

Lemma 3.6. We have $\left\|u_{n, i}-u_{0, i}-w_{0, i}\left(\cdot-y_{n}\right)\right\|_{H^{1}} \rightarrow 0$ for $i=1,2$. In addition, $\alpha=\gamma_{u_{0}}+\gamma_{w_{0}}$, $E\left(u_{0}\right)=e\left(\gamma_{u_{0}}\right), E_{\infty}\left(w_{0}\right)=e_{\infty}\left(\gamma_{w_{0}}\right)$ and $e(\alpha)=e\left(\gamma_{u_{0}}\right)+e_{\infty}\left(\gamma_{w_{0}}\right)$ where $\gamma_{w_{0}}=\left(\gamma_{w_{0}, 1}, \gamma_{w_{0}, 2}\right)$.

Proof. We first claim that

$$
\left\|u_{n}-u_{0}-w_{0}\left(\cdot-y_{n}\right)\right\|_{L^{2}} \rightarrow 0 .
$$

We prove (3.26) indirectly and suppose

$$
\lim _{n \rightarrow \infty}\left\|u_{n}-u_{0}-w_{0}\left(\cdot-y_{n}\right)\right\|_{L^{2}}>0 .
$$

By (3.25), one sees that $\gamma_{u_{0}}+\gamma_{w_{0}} \neq \alpha$. Applying the Brezis-Lieb Lemma and its proof (see, for instance, [43, Lemma 1.32]), for $2 \leq q \leq 2 N /(N-2)_{+}$and $1 \leq r \leq N /(N-2)_{+}$, we have

$$
\begin{aligned}
& \left\|u_{n, i}\right\|_{L^{q}}^{q}-\left\|u_{0, i}\right\|_{L^{q}}^{q}-\left\|u_{n, i}-u_{0, i}\right\|_{L^{q}}^{q}=o(1), \\
& \left.\left.\|\left(u_{n, i}-u_{0, i}\right)\left(\cdot+y_{n}\right)\right)\left\|_{L^{q}}^{q}-\right\| w_{0, i}\left\|_{L^{q}}^{q}-\right\|\left(u_{n, i}-u_{0, i}\right)\left(\cdot+y_{n}\right)\right)-w_{0, i} \|_{L^{q}}^{q}=o(1), \\
& \left\|\left|u_{n, i}\right|^{r}-\left|u_{0, i}\right|^{r}-\left|u_{n, i}-u_{0, i}\right|^{r}\right\|_{L^{2}}=o(1), \\
& \left\|\left|\left(u_{n, i}-u_{0, i}\right)\left(\cdot+y_{n}\right)\right|^{r}-\left|w_{0, i}\right|^{r}-\left|\left(u_{n, i}-u_{0, i}\right)\left(\cdot+y_{n}\right)-w_{0, i}\right|^{r}\right\|_{L^{2}}=o(1) .
\end{aligned}
$$

In particular,

$$
\left|u_{n, i}(x)\right|^{r}=\left|u_{0, i}(x)\right|^{r}+\left|w_{0, i}\left(x-y_{n}\right)\right|^{r}+\left|u_{n, i}(x)-u_{0, i}(x)-w_{0, i}\left(x-y_{n}\right)\right|^{r}+o(1) \quad \text { in } L^{2}\left(\mathbf{R}^{N}\right) .
$$

Combining this with (3.25) and $V_{j}(x) \rightarrow 0$ as $|x| \rightarrow \infty$, we obtain

$$
\begin{aligned}
& E\left(u_{n}\right)-E\left(u_{0}\right)-E\left(w_{0}\left(\cdot-y_{n}\right)\right)-E\left(u_{n}-u_{0}-w_{0}\left(\cdot-y_{n}\right)\right) \rightarrow 0, \\
& \delta_{0, i}:=\lim _{n \rightarrow \infty}\left\|u_{n, i}-u_{0, i}-w_{0, i}\left(\cdot-y_{n}\right)\right\|_{L^{2}}^{2}=\alpha_{i}-\gamma_{u_{0}, i}-\gamma_{w_{0}, i} .
\end{aligned}
$$

Here we notice that the assumption yields

$$
0 \leq \delta_{0, i}, \quad \delta_{0}:=\left(\delta_{0,1}, \delta_{0,2}\right) \neq(0,0) .
$$

Since $u_{n, i}(x)-u_{0, i}(x)-w_{0, i}\left(x-y_{n}\right) \rightarrow 0$ in $L_{\mathrm{loc}}^{q}\left(\mathbf{R}^{N}\right)$ for $2 \leq q<2 N /(N-2)_{+}$and $E\left(u_{n}-u_{0}-w_{0}\left(\cdot-y_{n}\right)\right)-E_{\infty}\left(u_{n}-u_{0}-w_{0}\left(\cdot-y_{n}\right)\right)=\sum_{i=1}^{2} \int_{\mathbf{R}^{N}} \frac{V_{i}(x)}{2}\left|u_{n, i}(x)-u_{0, i}(x)-w_{0, i}\left(x-y_{n}\right)\right|^{2} \mathrm{~d} x$, one observes that

$$
\int_{\mathbf{R}^{N}} V_{i}(x)\left|u_{n, i}(x)-u_{0, i}(x)-w_{0, i}\left(x-y_{n}\right)\right|^{2} \mathrm{~d} x \rightarrow 0 .
$$


Moreover, from the fact that $E_{\infty}$ is uniformly continuous on each bounded set and $\left|y_{n}\right| \rightarrow \infty$, it follows that

$$
\liminf _{n \rightarrow \infty} E\left(u_{n}-u_{0}-w_{0}\left(\cdot-y_{n}\right)\right) \geq e_{\infty}\left(\delta_{0}\right), \quad \liminf _{n \rightarrow \infty} E\left(w_{0}\left(\cdot-y_{n}\right)\right) \geq e_{\infty}\left(\gamma_{w_{0}}\right) .
$$

Thus (3.27), (3.29) and (3.30) yield

$$
e(\alpha) \geq e\left(\gamma_{u_{0}}\right)+e_{\infty}\left(\gamma_{w_{0}}\right)+e_{\infty}\left(\delta_{0}\right) .
$$

By noting that for $e_{\infty}(\alpha)$, the compactness of all minimizing sequences up to translations is equivalent to the strict subadditivity condition

$$
e_{\infty}(\alpha)<e_{\infty}(\gamma)+e_{\infty}(\alpha-\gamma) \text { for all } \gamma \in\left[0, \alpha_{1}\right] \times\left[0, \alpha_{2}\right] \text { with } \gamma \neq(0,0), \alpha .
$$

Therefore, by the result of [21] with (3.24) and (3.28), we have

$$
e_{\infty}\left(\gamma_{w_{0}}\right)+e_{\infty}\left(\delta_{0}\right)>e_{\infty}\left(\delta_{0}+\gamma_{w_{0}}\right)=e_{\infty}\left(\alpha-\gamma_{u_{0}}\right) .
$$

It follows from (3.31) that $e(\alpha)>e\left(\gamma_{u_{0}}\right)+e_{\infty}\left(\alpha-\gamma_{u_{0}}\right)$, however this contradicts Lemma 2.1(iii) and (3.26) holds.

By (3.26), one sees $\left\|u_{n, i}-u_{0, i}-w_{0, i}\left(\cdot-y_{n}\right)\right\|_{L^{q}} \rightarrow 0$ for each $2 \leq q<2 N /(N-2)_{+}$. Note also that (3.29) holds in this case. Therefore, as in the proof of Step 2, we get

$$
\begin{aligned}
e(\alpha)= & E\left(u_{n}\right)+o(1) \\
= & E\left(u_{0}\right)+E\left(w_{0}\left(\cdot-y_{n}\right)\right)+\frac{1}{2} \sum_{i=1}^{2}\left\|\nabla u_{n, i}-\nabla u_{0, i}-\nabla w_{0, i}\left(\cdot-y_{n}\right)\right\|_{L^{2}}^{2} \\
& +\frac{1}{2} \sum_{i=1}^{2} \int_{\mathbf{R}^{N}} V_{i}(x)\left|u_{n, i}(x)-u_{0, i}(x)-w_{0, i}\left(x-y_{n}\right)\right|^{2} \mathrm{~d} x+o(1) \\
\geq & e\left(\gamma_{u_{0}}\right)+e_{\infty}\left(\gamma_{w_{0}}\right)+\frac{1}{2} \sum_{i=1}^{2}\left\|\nabla u_{n, i}-\nabla u_{0, i}-\nabla w_{0, i}\left(\cdot-y_{n}\right)\right\|_{L^{2}}^{2}+o(1)
\end{aligned}
$$

which implies

$$
e(\alpha) \geq e\left(\gamma_{u_{0}}\right)+e_{\infty}\left(\gamma_{w_{0}}\right) .
$$

Moreover, it follows from (3.32) and Lemma 2.1(iii) that

$$
\begin{aligned}
& e(\alpha)=e\left(\gamma_{u_{0}}\right)+e\left(\gamma_{w_{0}}\right), \quad\left\|\nabla u_{n, i}-\nabla u_{0, i}-\nabla w_{0, i}\left(\cdot-y_{n}\right)\right\|_{L^{2}} \rightarrow 0(i=1,2), \\
& E\left(u_{0}\right)=e\left(\gamma_{u_{0}}\right), \quad E_{\infty}\left(w_{0}\right)=e_{\infty}\left(\gamma_{w_{0}}\right) .
\end{aligned}
$$

Hence, by combining this with (3.26), Lemma 3.6 holds.

To proceed, we recall (3.22), $\lambda_{n, i} \rightarrow \lambda_{0, i}$, and $u_{n} \rightarrow u_{0}$ and $u_{n}\left(\cdot+y_{n}\right) \rightarrow w_{0}$ weakly in $H$. Hence, $u_{0}$ (resp. $w_{0}$ ) is a nonnegative solution of (3.1) (resp. (3.3)). Our next task is to show $0<\lambda_{0, i}$ for $i=1,2$ :

Lemma 3.7. Suppose (p2) in addition to (V1) and (p1). Then $\lambda_{0, i}>0(i=1,2)$ hold.

Proof. We remark that by Lemma 3.6 and $\alpha_{i}>0(i=1,2)$, for each $i=1,2$, either $u_{0, i} \not \equiv 0$ or $w_{0, i} \not \equiv 0$. In what follows, we only show that $\lambda_{0,1}>0$ holds when $u_{0,1} \not \equiv 0$, but other cases can be treated similarly.

Assume $u_{0,1} \not \equiv 0$. Then $u_{0,1}>0$ in $\mathbf{R}^{N}$ by the strong maximum principle. If $\lambda_{0,1} \leq 0$, then by $u_{0, i} \geq 0, V_{i}(x) \leq 0$ and $\beta>0$, it follows from (3.1) that

$$
-\Delta u_{0,1} \geq \mu_{1} u_{0,1}^{2 p_{1}+1} \quad \text { in } \mathbf{R}^{N} .
$$

However, this inequality does not have any positive solution for $2 p_{1}+1 \leq N /(N-2)$ when $N \geq 3$. See, for instance, [39, Theorem 8.4]. When $N=1$, it is easy to see that the inequality admits no positive solution. If $N=2$, since $u_{0,1}$ is bounded by elliptic regularity and $u_{0,1}(x) \rightarrow 0$ as $|x| \rightarrow \infty$, [37, Theorem 29] yields $u_{0, i} \equiv 0$. Therefore, we have a contradiction and $\lambda_{0,1}>0$ holds provided $u_{0,1} \not \equiv 0$. 
From Lemma 3.7, without loss of generality, we may assume (3.2): $0<\lambda_{0,1} \leq \lambda_{0,2}$. Thus, we note that Lemma 3.1 is applicable to $u_{0}$ and $w_{0}$.

Now under (V1) and (p1)-(p2), we derive a contradiction and complete the proof of Theorem 1.1:

Proof of Theorem 1.1. By Lemma 3.6, we have

$$
\begin{aligned}
& e(\alpha)=e\left(\gamma_{u_{0}}\right)+e_{\infty}\left(\gamma_{w_{0}}\right), \quad E\left(u_{0}\right)=e\left(\gamma_{u_{0}}\right), \quad E_{\infty}\left(w_{0}\right)=e_{\infty}\left(\gamma_{w_{0}}\right), \quad w_{0} \neq(0,0), \\
& \left.u_{0} \text { (resp. } w_{0}\right) \text { is a nonnegative solution of }(3.1) \text { (resp. (3.3)) with (3.2). }
\end{aligned}
$$

In what follows, we will find a $u \in M(\alpha)$ such that

$$
E(u)<e\left(\gamma_{u_{0}}\right)+e_{\infty}\left(\gamma_{w_{0}}\right),
$$

which leads a contradiction and we conclude that $\left(u_{n}\right)$ has a strongly convergent subsequence in $H$.

To show (3.34), set

$$
w_{n, i}(x):=w_{0, i}\left(x-n \mathbf{e}_{1}\right), \quad \tau_{n, i}:=\frac{\sqrt{\alpha_{i}}}{\left\|u_{0, i}+w_{n, i}\right\|_{L^{2}}}, \quad \kappa_{n, i}:=\left\langle u_{0, i}, w_{n, i}\right\rangle_{L^{2}} .
$$

Remark that

$$
\left(\tau_{n, 1}\left[u_{0,1}+w_{n, 1}\right], \tau_{n, 2}\left[u_{0,2}+w_{n, 2}\right]\right) \in M(\alpha), \quad 0 \leq \kappa_{n, i} \rightarrow 0 .
$$

Our goal is to prove

$$
E\left(\tau_{n, 1}\left[u_{0,1}+w_{n, 1}\right], \tau_{n, 2}\left[u_{0,2}+w_{n, 2}\right]\right)<e\left(\gamma_{u_{0}}\right)+e_{\infty}\left(\gamma_{w_{0}}\right)
$$

for sufficiently large $n$. We divide our proof into several steps.

Step 1: In the following cases, (3.35) holds: (i) $u_{0}=(0,0)$, (ii) $u_{0}=\left(u_{0,1}, 0\right)$ and $w_{0}=\left(0, w_{0,2}\right)$, (iii) $u_{0}=\left(0, u_{0,2}\right)$ and $w_{0}=\left(w_{0,1}, 0\right)$.

In each case, we have $\kappa_{n, 1}=\kappa_{n, 2}=0$ and $\tau_{n, 1}=1=\tau_{n, 2}$. When $u_{0}=(0,0)$, notice that $e(\alpha)=$ $e_{\infty}\left(\gamma_{w_{0}}\right)$ thanks to $(3.33)$ and that

$$
\begin{aligned}
E\left(w_{n, 1}, w_{n, 2}\right) & =E_{\infty}\left(w_{n, 1}, w_{n, 2}\right)+\frac{1}{2} \int_{\mathbf{R}^{N}} V_{1}(x) w_{n, 1}^{2}+V_{2}(x) w_{n, 2}^{2} \mathrm{~d} x \\
& =e(\alpha)+\frac{1}{2} \int_{\mathbf{R}^{N}} V_{1}(x) w_{n, 1}^{2}+V_{2}(x) w_{n, 2}^{2} \mathrm{~d} x .
\end{aligned}
$$

Since $V_{j}(x) \leq 0(j=1,2), w_{j}>0(j=1,2)$ and either $V_{1}(x) \not \equiv 0$ or $V_{2}(x) \not \equiv 0$, we get $E\left(w_{n, 1}, w_{n, 2}\right)<$ $e(\alpha)$ and (3.35) holds.

In the case $u_{0}=\left(u_{0,1}, 0\right)$ and $w_{0}=\left(0, w_{0,2}\right)$, it follows from $u_{0,1}, w_{0,2}>0$ and $\beta>0$ that

$$
E\left(u_{0,1}, w_{n, 2}\right) \leq E\left(u_{0,1}, 0\right)+E_{\infty}\left(0, w_{n, 2}\right)-\frac{\beta}{p_{3}+1} \int_{\mathbf{R}^{N}} u_{0,1}^{p_{3}+1} w_{n, 2}^{p_{3}+1} \mathrm{~d} x<e\left(\gamma_{u_{0}}\right)+e_{\infty}\left(\gamma_{w_{0}}\right)=e(\alpha) .
$$

Hence, (3.35) holds. The case $u_{0}=\left(0, u_{0,2}\right)$ and $w_{0}=\left(w_{0,1}, 0\right)$ can be treated similarly. Thus Step 1 holds.

From Step 1, we may suppose either $u_{0,1}, w_{0,1}>0$ or $u_{0,2}, w_{0,2}>0$, hence we may assume that

$$
\text { either } \kappa_{n, 1}>0 \text { or } 0=\kappa_{n, 1}<\kappa_{n, 2} \text {. }
$$

Step 2: (i) If $\kappa_{n, 1}>0$, then for each $\lambda<\lambda_{0,1}$, there exist $c>0$, which is independent of $\lambda$, and $0<C_{\lambda}$ such that

$$
c(1+n)^{-(N-1) / 2} \exp \left(-\sqrt{\lambda_{0,1}} n\right) \leq \kappa_{n, 1} \leq C_{\lambda} \exp (-\sqrt{\lambda} n) \text { for all } n \geq 1 .
$$

Moreover, if $\lambda_{0,1}=\lambda_{0,2}$ and $\kappa_{n, 2}>0$, then $\kappa_{n, 2}$ also satisfies (3.37). If $\lambda_{0,1}<\lambda_{0,2}$, then there exist $\theta_{1}>1$ and $C_{0}>0$ such that

$$
\kappa_{n, 2} \leq C_{0} \kappa_{n, 1}^{\theta_{1}} \text { for all } n \geq 1
$$


(ii) Suppose $\kappa_{n, 1}=0$ and $\kappa_{n, 2}>0$. When either $p_{3} \geq 1$ or else $0<p_{3}<1$ and $\lambda_{0,3}=\left(1+p_{3}\right)^{2} \lambda_{0,1} /(1-$ $\left.p_{3}\right)^{2}>\lambda_{0,2}$, for every $\lambda<\lambda_{0,2}$, there exist $c>0$, which is independent of $\lambda$, and $0<C_{\lambda}$ such that

$$
c(1+n)^{-(N-1) / 2} \exp \left(-\sqrt{\lambda_{0,2}} n\right) \leq \kappa_{n, 2} \leq C_{\lambda} \exp (-\sqrt{\lambda} n) \quad \text { for all } n \geq 1 .
$$

When $0<p_{3}<1$ and $\lambda_{0,3} \leq \lambda_{0,2}$, for every $\lambda_{1}<\lambda_{0,3}<\lambda_{2}$ there exist $C_{\lambda_{1}}, C_{\lambda_{2}}>0$ such that

$$
C_{\lambda_{2}} \exp \left(-\sqrt{\lambda_{2}} n\right) \leq \kappa_{n, 2} \leq C_{\lambda_{1}} \exp \left(-\sqrt{\lambda_{1}} n\right) \text { for all } n \geq 1 \text {. }
$$

(i) By $\kappa_{n, 1}>0$, one has $u_{0,1}, w_{0,1}>0$. From Lemma 3.1 (i), for any $\lambda<\lambda_{0,1}$, it follows that

$$
\begin{aligned}
& u_{0,1}(x) \exp (\sqrt{\lambda}|x|)+w_{0,1}(x) \exp \left(\sqrt{\frac{\lambda+\lambda_{0,1}}{2}}|x|\right) \rightarrow 0 \quad \text { as }|x| \rightarrow \infty, \\
& c(1+|x|)^{-(N-1) / 2} \exp \left(-\sqrt{\lambda_{0,1}}|x|\right) \leq u_{0,1}(x), \quad c \exp \left(-\sqrt{\lambda_{0,1}+1}|x|\right) \leq w_{0,1}(x) .
\end{aligned}
$$

Thus, Lemma 3.3 asserts

$$
\exp (\sqrt{\lambda} n) \kappa_{n, 1} \rightarrow 0
$$

and

$$
\begin{aligned}
& (1+n)^{(N-1) / 2} \exp \left(\sqrt{\lambda_{0,1}} n\right) \kappa_{n, 1} \\
\geq & c^{2}(1+n)^{(N-1) / 2} \exp \left(\sqrt{\lambda_{0,1}} n\right) \\
& \quad \times \int_{\mathbf{R}^{N}}\left(1+\left|y-n \mathbf{e}_{1}\right|\right)^{-(N-1) / 2} \exp \left(-\sqrt{\lambda_{0,1}}\left|y-n \mathbf{e}_{1}\right|\right) \exp \left(-\sqrt{\lambda_{0,1}+1}|y|\right) \mathrm{d} y \\
\rightarrow & c^{2} \int_{\mathbf{R}^{N}} \exp \left(-\sqrt{\lambda_{0,1}+1}|y|\right) \exp \left(\sqrt{\lambda_{0,1}} \mathbf{e}_{1} \cdot y\right) \mathrm{d} y>0 .
\end{aligned}
$$

Hence, (3.37) holds.

If $\lambda_{0,1}=\lambda_{0,2}$ and $\kappa_{n, 2}>0$ hold, then from Lemma 3.1 (ii) and (iii), we observe that $u_{0,2}$ satisfies the decay estimate in Lemma 3.1 (i). Thus, we can argue as in the above and see that $\kappa_{n, 2}$ satisfies (3.37).

Finally, if $\lambda_{0,1}<\lambda_{0,2}$, by Lemma 3.1 (ii) and (iii), one can find an $\delta_{0}>0$ such that

$$
0 \leq u_{0,2}(x) \leq C \exp \left(-\sqrt{\lambda_{0,1}+2 \delta_{0}}|x|\right), \quad w_{0,2}(x) \leq C \exp \left(-\sqrt{\lambda_{0,1}+3 \delta_{0}}|x|\right) .
$$

Hence, Lemma 3.3 yields

$$
\exp \left(\sqrt{\lambda_{0,1}+\delta_{0}} n\right) \kappa_{n, 2} \rightarrow 0
$$

Thus, (3.38) holds for some $\theta_{1}>1$ and $C_{0}>0$ due to (3.37).

(ii) By $\kappa_{n, 1}=0$, remark that either $u_{0}=\left(0, u_{0,2}\right)$ or $w_{0}=\left(0, w_{0,2}\right)$. Since the arguments for both cases are similar, we only deal with the case $u_{0}=\left(0, u_{0,2}\right)$. From $\kappa_{n, 2}>0$ and $\alpha=\gamma_{u_{0}}+\gamma_{w_{0}}$, we have $w_{0}=\left(w_{0,1}, w_{0,2}\right)$ with $w_{0, j}>0(j=1,2)$. Moreover, by Lemma 3.1 (ii), for every $\lambda \in\left(0, \lambda_{0,2}\right)$ there exists a $C_{\lambda}>0$ such that

$$
c_{0}(1+|x|)^{-(N-1) / 2} \exp \left(-\sqrt{\lambda_{0,2}}|x|\right) \leq u_{0,2}(x) \leq C_{\lambda} \exp (-\sqrt{\lambda}|x|) .
$$

When either $p_{3} \geq 1$ or else $0<p_{3}<1$ and $\lambda_{0,3}=\left(1+p_{3}\right)^{2} \lambda_{0,1} /\left(1-p_{3}\right)^{2}>\lambda_{0,2}$, by Lemma 3.1 (iii), $w_{0,2}$ satisfies the same estimate to (3.41):

$$
c_{0}(1+|x|)^{-(N-1) / 2} \exp \left(-\sqrt{\lambda_{0,2}}|x|\right) \leq w_{0,2}(x) \leq C_{\lambda} \exp (-\sqrt{\lambda}|x|)
$$

for every $\lambda \in\left(0, \lambda_{0,2}\right)$. Hence, as in (i), we can prove (3.39).

Next, suppose $0<p_{3}<1$ and $\lambda_{0,3} \leq \lambda_{0,2}$. Lemma 3.1 (iii) gives

$$
C_{\lambda_{2}} \exp \left(-\sqrt{\lambda_{2}}|x|\right) \leq w_{0,2}(x) \leq C_{\lambda_{1}} \exp \left(-\sqrt{\lambda_{1}}|x|\right)
$$

for every $\lambda_{1} \in\left(0, \lambda_{0,3}\right)$ and $\lambda_{2} \in\left(\lambda_{0,3}, \infty\right)$. The rest of arguments is similar to the above and we can prove $(3.40)$. 
Step 3: Estimate of $E\left(\tau_{n, 1}\left[u_{0,1}+w_{n, 1}\right], \tau_{n, 2}\left[u_{0,2}+w_{n, 2}\right]\right)$.

Since $\alpha_{i}=\left\|u_{0, i}\right\|_{L^{2}}^{2}+\left\|w_{0, i}\right\|_{L^{2}}^{2}$, we have

$$
\left\|u_{0, i}+w_{n, i}\right\|_{L^{2}}^{2}=\alpha_{i}+2 \kappa_{n, i}
$$

Hence,

$$
\tau_{n, i}^{2}=\frac{\alpha_{i}}{\alpha_{i}+2 \kappa_{n, i}}=\frac{1}{1+2 \kappa_{n, i} / \alpha_{i}}=1-\frac{2 \kappa_{n, i}}{\alpha_{i}}+O\left(\kappa_{n, i}^{2}\right), \quad \tau_{n, i}=1-\frac{\kappa_{n, i}}{\alpha_{i}}+O\left(\kappa_{n, i}^{2}\right)
$$

and $\tau_{n, i}=1$ if $\kappa_{n, i}=0$.

Recalling the notation in (2.1) and noting

$$
\begin{aligned}
& E\left(\tau_{n, 1}\left[u_{0,1}+w_{n, 1}\right], \tau_{n, 2}\left[u_{0,2}+w_{n, 2}\right]\right) \\
= & \sum_{i=1}^{2} \frac{\tau_{n, i}^{2}}{2}\left\|u_{0, i}+w_{n, i}\right\|_{V_{i}}^{2}-\int_{\mathbf{R}^{N}} \sum_{i=1}^{2} \frac{\mu_{i} \tau_{n, i}^{2 p_{i}+2}}{2 p_{i}+2}\left(u_{0, i}+w_{n, i}\right)^{2 p_{i}+2} \mathrm{~d} x \\
& -\int_{\mathbf{R}^{N}} \frac{\beta \tau_{n, 1}^{p_{3}+1} \tau_{n, 2}^{p_{3}+1}}{p_{3}+1}\left(u_{0,1}+w_{n, 1}\right)^{p_{3}+1}\left(u_{0,2}+w_{n, 2}\right)^{p_{3}+1} \mathrm{~d} x,
\end{aligned}
$$

we compute each term.

First, it follows from (3.42) and $\left\langle u_{0, i}, w_{n, i}\right\rangle_{V_{i}} \rightarrow 0$ as $n \rightarrow \infty$ that

$$
\begin{aligned}
& \frac{\tau_{n, i}^{2}}{2}\left\|u_{0, i}+w_{n, i}\right\|_{V_{i}}^{2} \\
= & \frac{1}{2}\left(1-\frac{2 \kappa_{n, i}}{\alpha_{i}}+O\left(\kappa_{n, i}^{2}\right)\right)\left(\left\|u_{0, i}\right\|_{V_{i}}^{2}+\left\|w_{n, i}\right\|_{V_{i}}^{2}+2\left\langle u_{0, i}, w_{n, i}\right\rangle_{V_{i}}\right) \\
= & \frac{1}{2}\left(\left\|u_{0, i}\right\|_{V_{i}}^{2}+\left\|w_{n, i}\right\|_{V_{i}}^{2}+2\left\langle u_{0, i}, w_{n, i}\right\rangle_{V_{i}}\right)-\frac{\kappa_{n, i}}{\alpha_{i}}\left(\left\|u_{0, i}\right\|_{V_{i}}^{2}+\left\|w_{n, i}\right\|_{V_{i}}^{2}\right) \\
& +O\left(\kappa_{n, i}^{2}\right)+O\left(\kappa_{n, i}\left\langle u_{0, i}, w_{n, i}\right\rangle_{V_{i}}\right) \\
= & \frac{1}{2}\left(\left\|u_{0, i}\right\|_{V_{i}}^{2}+\left\|\nabla w_{0, i}\right\|_{L^{2}}^{2}+2\left\langle u_{0, i}, w_{n, i}\right\rangle_{V_{i}}\right)-\frac{\kappa_{n, i}}{\alpha_{i}}\left(\left\|u_{0, i}\right\|_{V_{i}}^{2}+\left\|\nabla w_{0, i}\right\|_{L^{2}}^{2}\right) \\
& +\left(\frac{1}{2}-\frac{\kappa_{n, i}}{\alpha_{i}}\right) \int_{\mathbf{R}^{N}} V_{i}(x) w_{n, i}^{2} \mathrm{~d} x+O\left(\kappa_{n, i}^{2}\right)+O\left(\kappa_{n, i}\left\langle u_{0, i}, w_{n, i}\right\rangle_{V_{i}}\right) .
\end{aligned}
$$

Since $u_{0}$ is a solution of (3.1), one sees

$$
\begin{aligned}
& \left\langle u_{0,1}, w_{n, 1}\right\rangle_{V_{1}}=-\lambda_{0,1} \kappa_{n, 1}+\int_{\mathbf{R}^{N}} \mu_{1} u_{0,1}^{2 p_{1}+1} w_{n, 1}+\beta u_{0,1}^{p_{3}} u_{0,2}^{p_{3}+1} w_{n, 1} \mathrm{~d} x, \\
& \left\langle u_{0,2}, w_{n, 2}\right\rangle_{V_{2}}=-\lambda_{0,2} \kappa_{n, 2}+\int_{\mathbf{R}^{N}} \mu_{2} u_{0,2}^{2 p_{2}+1} w_{n, 2}+\beta u_{0,1}^{p_{3}+1} u_{0,2}^{p_{3}} w_{n, 2} \mathrm{~d} x .
\end{aligned}
$$

By Lemma 3.1, we notice that

$$
u_{0,1}^{2 p_{1}+1}(x)+u_{0,1}^{p_{3}}(x) u_{0,2}^{p_{3}+1}(x)+\mu_{2} u_{0,2}^{2 p_{2}+1}(x)+\beta u_{0,1}^{p_{3}+1}(x) u_{0,2}^{p_{3}}(x) \leq C \exp \left(-\sqrt{\lambda_{0,1}}|x|\right) .
$$

From Lemma 3.3 it follows that

$$
\int_{\mathbf{R}^{N}} \mu_{1} u_{0,1}^{2 p_{1}+1} w_{n, 1}+\beta u_{0,1}^{p_{3}} u_{0,2}^{p_{3}+1} w_{n, 1}+\mu_{2} u_{0,2}^{2 p_{2}+1} w_{n, 2}+\beta u_{0,1}^{p_{3}+1} u_{0,2}^{p_{3}} w_{n, 2} \mathrm{~d} x \leq C_{0} \exp \left(-\sqrt{\frac{\lambda_{0,1}}{2} n}\right) .
$$

Noting Step 2, we may find a $\theta_{2} \in(1,2)$ such that

$$
\kappa_{n, i}\left\langle u_{0, i}, w_{n, i}\right\rangle_{V_{i}}=o\left(\kappa_{n, i}^{\theta_{2}}\right) \quad \text { for } i=1,2
$$


Since $\kappa_{n, i} \rightarrow 0$, we may assume $1 / 2-\kappa_{n, i} / \alpha_{i} \geq 0$ and it follows from $V_{i}(x) \leq 0,(3.44)-(3.46)$ and $1<\theta_{2}<2$ that

$$
\begin{aligned}
& \frac{\tau_{n, 1}^{2}}{2}\left\|u_{0,1}+w_{n, 1}\right\|_{V_{1}}^{2} \\
\leq & \frac{1}{2}\left(\left\|u_{0,1}\right\|_{V_{1}}^{2}+\left\|\nabla w_{0,1}\right\|_{L^{2}}^{2}\right)-\lambda_{0,1} \kappa_{n, 1}+\int_{\mathbf{R}^{N}} \mu_{1} u_{0,1}^{2 p_{1}+1} w_{n, 1}+\beta u_{0,1}^{p_{3}} u_{0,2}^{p_{3}+1} w_{n, 1} \mathrm{~d} x \\
& -\frac{\kappa_{n, 1}}{\alpha_{1}}\left(\left\|u_{0,1}\right\|_{V_{1}}^{2}+\left\|\nabla w_{0,1}\right\|_{L^{2}}^{2}\right)+o\left(\kappa_{n, 1}^{\theta_{2}}\right)
\end{aligned}
$$

and

$$
\begin{aligned}
& \frac{\tau_{n, 2}^{2}}{2}\left\|u_{0,2}+w_{n, 2}\right\|_{V_{2}}^{2} \\
\leq & \frac{1}{2}\left(\left\|u_{0,2}\right\|_{V_{2}}^{2}+\left\|\nabla w_{0,2}\right\|_{L^{2}}^{2}\right)-\lambda_{0,2} \kappa_{n, 2}+\int_{\mathbf{R}^{N}} \mu_{2} u_{0,2}^{2 p_{2}+1} w_{n, 2}+\beta u_{0,1}^{p_{3}+1} u_{0,2}^{p_{3}} w_{n, 2} \mathrm{~d} x \\
& -\frac{\kappa_{n, 2}}{\alpha_{2}}\left(\left\|u_{0,2}\right\|_{V_{2}}^{2}+\left\|\nabla w_{0,2}\right\|_{L^{2}}^{2}\right)+o\left(\kappa_{n, 2}^{\theta_{2}}\right) .
\end{aligned}
$$

Next, it follows from (3.42) and Lemma 3.4 that

$$
\begin{aligned}
& -\frac{\mu_{1} \tau_{n, 1}^{2 p_{1}+2}}{2 p_{1}+2} \int_{\mathbf{R}^{N}}\left(u_{0,1}+w_{n, 1}\right)^{2 p_{1}+2} \mathrm{~d} x \\
\leq & -\frac{\mu_{1}}{2 p_{1}+2}\left(1-\frac{2 p_{1}+2}{\alpha_{1}} \kappa_{n, 1}\right) \\
& \times \int_{\mathbf{R}^{N}} u_{0,1}^{2 p_{1}+2}+w_{n, 1}^{2 p_{1}+2}+\left(2 p_{1}+2\right)\left(u_{0,1}^{2 p_{1}+1} w_{n, 1}+u_{0,1} w_{n, 1}^{2 p_{1}+1}\right)-C_{p_{1}} u_{0,1}^{p_{1}+1} w_{n, 1}^{p_{1}+1} \mathrm{~d} x+O\left(\kappa_{n, 1}^{2}\right) .
\end{aligned}
$$

Thanks to $1<p_{1}+1$ and Lemma 3.1, we may find an $\eta_{1}>0$ such that

$$
u_{0,1}^{p_{1}+1}(x)+w_{0,1}^{p_{1}+1}(x) \leq C \exp \left(-\sqrt{\lambda_{0,1}+\eta_{1}}|x|\right) .
$$

Therefore, exploiting Lemma 3.3, we can find a $\theta_{3}=\theta_{3}\left(p_{1}\right) \in(1,2)$ such that

$$
\kappa_{n, 1} \int_{\mathbf{R}^{N}} u_{0,1}^{2 p_{1}+1} w_{n, 1}+u_{0,1} w_{n, 1}^{2 p_{1}+1} \mathrm{~d} x+\int_{\mathbf{R}^{N}} u_{0,1}^{p_{1}+1} w_{n, 1}^{p_{1}+1} \mathrm{~d} x=o\left(\kappa_{n, 1}^{\theta_{3}}\right) .
$$

Hence,

$$
\begin{aligned}
& -\frac{\mu_{1} \tau_{n, 1}^{2 p_{1}+2}}{2 p_{1}+2} \int_{\mathbf{R}^{N}}\left(u_{0,1}+w_{n, 1}\right)^{2 p_{1}+2} \mathrm{~d} x \\
\leq & -\frac{\mu_{1}}{2 p_{1}+2} \int_{\mathbf{R}^{N}} u_{0,1}^{2 p_{1}+2}+w_{0,1}^{2 p_{1}+2} \mathrm{~d} x-\mu_{1} \int_{\mathbf{R}^{N}} u_{0,1}^{2 p_{1}+1} w_{n, 1}+u_{0,1} w_{n, 1}^{2 p_{1}+1} \mathrm{~d} x \\
& +\frac{\kappa_{n, 1}}{\alpha_{1}} \mu_{1} \int_{\mathbf{R}^{N}} u_{0,1}^{2 p_{1}+2}+w_{0,1}^{2 p_{1}+2} \mathrm{~d} x+o\left(\kappa_{n, 1}^{\theta_{3}}\right) .
\end{aligned}
$$

Similarly, we have

$$
\begin{aligned}
& -\frac{\mu_{2} \tau_{n, 2}^{2 p_{2}+2}}{2 p_{2}+2} \int_{\mathbf{R}^{N}}\left(u_{0,2}+w_{n, 2}\right)^{2 p_{2}+2} \mathrm{~d} x \\
\leq & -\frac{\mu_{2}}{2 p_{2}+2} \int_{\mathbf{R}^{N}} u_{0,2}^{2 p_{2}+2}+w_{0,2}^{2 p_{2}+2} \mathrm{~d} x-\mu_{2} \int_{\mathbf{R}^{N}} u_{0,2}^{2 p_{2}+1} w_{n, 2}+u_{0,2} w_{n, 2}^{2 p_{2}+1} \mathrm{~d} x \\
& +\frac{\kappa_{n, 2}}{\alpha_{2}} \mu_{2} \int_{\mathbf{R}^{N}} u_{0,2}^{2 p_{2}+2}+w_{0,2}^{2 p_{2}+2} \mathrm{~d} x+o\left(\kappa_{n, 2}^{\theta_{4}}\right)
\end{aligned}
$$

for some $\theta_{4}=\theta_{4}\left(p_{2}\right) \in(1,2)$. 
On the other hand, choosing $\eta=p_{3} / 2$ in Lemma 3.4 (ii), it follows from (3.42) that

$$
\begin{aligned}
& -\frac{\beta \tau_{n, 1}^{p_{3}+1} \tau_{n, 2}^{p_{3}+1}}{p_{3}+1} \int_{\mathbf{R}^{N}}\left(u_{0,1}+w_{n, 1}\right)^{p_{3}+1}\left(u_{0,2}+w_{n, 2}\right)^{p_{3}+1} \mathrm{~d} x \\
\leq & -\frac{\beta}{p_{3}+1}\left(1-\frac{p_{3}+1}{\alpha_{1}} \kappa_{n, 1}\right)\left(1-\frac{p_{3}+1}{\alpha_{2}} \kappa_{n, 2}\right) \\
& \times \int_{\mathbf{R}^{N}} u_{0,1}^{p_{3}+1} u_{0,2}^{p_{3}+1}+w_{0,1}^{p_{3}+1} w_{0,2}^{p_{3}+1}+\left(p_{3}+1\right)\left(u_{0,1}^{p_{3}} u_{0,2}^{p_{3}+1} w_{n, 1}+u_{0,1}^{p_{3}+1} u_{0,2}^{p_{3}} w_{n, 2}+u_{0,2} w_{n, 1}^{p_{3}+1} w_{n, 2}^{p_{3}}\right) \mathrm{d} x \\
& +\frac{\beta}{p_{3}+1}\left(1-\frac{p_{3}+1}{\alpha_{1}} \kappa_{n, 1}\right)\left(1-\frac{p_{3}+1}{\alpha_{2}} \kappa_{n, 2}\right) C_{p_{3}} \int_{\mathbf{R}^{N}} u_{0,1}^{p_{3} / 2} u_{0,2}^{p_{3}+1} w_{n, 1}^{1+p_{3} / 2}+u_{0,1}^{1+p_{3} / 2} w_{n, 1}^{p_{3} / 2} w_{n, 2}^{p_{3}+1} \mathrm{~d} x \\
& +O\left(\kappa_{n, 1}^{2}+\kappa_{n, 2}^{2}\right) .
\end{aligned}
$$

As in the above, if $\kappa_{n, 1}>0$, then by Lemma 3.1 and Step 2, we may find a $\theta_{5} \in(1,2)$ such that

$$
\begin{aligned}
o\left(\kappa_{n, 1}^{\theta_{5}}\right)= & \sum_{i=1}^{2} \kappa_{n, i} \int_{\mathbf{R}^{N}} u_{0,1}^{p_{3}} u_{0,2}^{p_{3}+1} w_{n, 1}+u_{0,1}^{p_{3}+1} u_{0,2}^{p_{3}} w_{n, 2}+u_{0,2} w_{n, 1}^{p_{3}+1} w_{n, 2}^{p_{3}} \mathrm{~d} x \\
& +\int_{\mathbf{R}^{N}} u_{0,1}^{p_{3} / 2} u_{0,2}^{p_{3}+1} w_{n, 1}^{1+p_{3} / 2}+u_{0,1}^{1+p_{3} / 2} w_{n, 1}^{p_{3} / 2} w_{n, 2}^{p_{3}+1} \mathrm{~d} x .
\end{aligned}
$$

On the other hand, if $\kappa_{n, 1}=0<\kappa_{n, 2}$, then $u_{0,1}^{p_{3} / 2} u_{0,2}^{p_{3}+1} w_{n, 1}^{1+p_{3} / 2} \equiv 0 \equiv u_{0,1}^{1+p_{3} / 2} w_{n, 1}^{p_{3} / 2} w_{n, 2}^{p_{3}+1}$. Therefore, if $\kappa_{n, 1}=0<\kappa_{n, 2}$, then for some $\theta_{5} \in(1,2)$, one has

$$
\begin{aligned}
o\left(\kappa_{n, 2}^{\theta_{5}}\right)= & \sum_{i=1}^{2} \kappa_{n, i} \int_{\mathbf{R}^{N}} u_{0,1}^{p_{3}} u_{0,2}^{p_{3}+1} w_{n, 1}+u_{0,1}^{p_{3}+1} u_{0,2}^{p_{3}} w_{n, 2}+u_{0,2} w_{n, 1}^{p_{3}+1} w_{n, 2}^{p_{3}} \mathrm{~d} x \\
& +\int_{\mathbf{R}^{N}} u_{0,1}^{p_{3} / 2} u_{0,2}^{p_{3}+1} w_{n, 1}^{1+p_{3} / 2}+u_{0,1}^{1+\eta} w_{n, 1}^{p_{3} / 2} w_{n, 2}^{p_{3}+1} \mathrm{~d} x .
\end{aligned}
$$

Thus, in both cases, we infer that

$$
\begin{aligned}
& -\frac{\beta \tau_{n, 1}^{p_{3}+1} \tau_{n, 2}^{p_{3}+1}}{p_{3}+1} \int_{\mathbf{R}^{N}}\left(u_{0,1}+w_{n, 1}\right)^{p_{3}+1}\left(u_{0,2}+w_{n, 2}\right)^{p_{3}+1} \mathrm{~d} x \\
\leq & -\frac{\beta}{p_{3}+1} \int_{\mathbf{R}^{N}} u_{0,1}^{p_{3}+1} u_{0,2}^{p_{3}+1}+w_{0,1}^{p_{3}+1} w_{0,2}^{p_{3}+1} \mathrm{~d} x \\
& -\beta \int_{\mathbf{R}^{N}} u_{0,1}^{p_{3}} u_{0,2}^{p_{3}+1} w_{n, 1}+u_{0,1}^{p_{3}+1} u_{0,2}^{p_{3}} w_{n, 2}+u_{0,2} w_{n, 1}^{p_{3}+1} w_{n, 2}^{p_{3}} \mathrm{~d} x \\
& +\beta\left(\frac{\kappa_{n, 1}}{\alpha_{1}}+\frac{\kappa_{n, 2}}{\alpha_{2}}\right) \int_{\mathbf{R}^{N}} u_{0,1}^{p_{3}+1} u_{0,2}^{p_{3}+1}+w_{0,1}^{p_{3}+1} w_{0,2}^{p_{3}+1} \mathrm{~d} x+o\left(\kappa_{n, 1}^{\theta_{5}}+\kappa_{n, 2}^{\theta_{5}}\right) .
\end{aligned}
$$

From (3.38), (3.43) and (3.47)-(3.51), setting $\theta_{0}:=\min \left\{\theta_{j} \mid 1 \leq j \leq 5\right\} \in(1,2)$, we have

$$
\begin{aligned}
& E\left(\tau_{n, 1}\left[u_{0,1}+w_{n, 1}\right], \tau_{n, 2}\left[u_{0,2}+w_{n, 2}\right]\right) \\
\leq & E\left(u_{0}\right)+E_{\infty}\left(w_{0}\right)-\sum_{i=1}^{2} \frac{\kappa_{n, i}}{\alpha_{i}}\left(\lambda_{0, i} \alpha_{i}+\left\|u_{0, i}\right\|_{V_{i}}^{2}+\left\|\nabla w_{0, i}\right\|_{L^{2}}^{2}-\mu_{i} \int_{\mathbf{R}^{N}} u_{0, i}^{2 p_{i}+2}+w_{0, i}^{2 p_{i}+2} \mathrm{~d} x\right) \\
& -\int_{\mathbf{R}^{N}} \mu_{1} u_{0,1} w_{n, 1}^{2 p_{1}+1}+\mu_{2} u_{0,2} w_{n, 2}^{2 p_{2}+1}+\beta u_{0,2} w_{n, 1}^{p_{3}+1} w_{n, 2}^{p_{3}} \mathrm{~d} x \\
& +\beta\left(\frac{\kappa_{n, 1}}{\alpha_{1}}+\frac{\kappa_{n, 2}}{\alpha_{2}}\right) \int_{\mathbf{R}^{N}} u_{0,1}^{p_{3}+1} u_{0,2}^{p_{3}+1}+w_{0,1}^{p_{3}+1} w_{0,2}^{p_{3}+1} \mathrm{~d} x+ \begin{cases}o\left(\kappa_{n, 1}^{\theta_{0}}\right) & \text { if } \kappa_{n, 1}>0, \\
o\left(\kappa_{n, 2}^{\theta_{0}}\right) & \text { if } \kappa_{n, 1}=0<\kappa_{n, 2} .\end{cases}
\end{aligned}
$$


By (3.1) and (3.3), note that

$$
\begin{gathered}
\left\|u_{0,1}\right\|_{V_{1}}^{2}=-\lambda_{0,1}\left\|u_{0,1}\right\|_{L^{2}}^{2}+\int_{\mathbf{R}^{N}} \mu_{1} u_{0,1}^{2 p_{1}+2}+\beta u_{0,1}^{p_{3}+1} u_{0,2}^{p_{3}+1} \mathrm{~d} x, \\
\left\|u_{0,2}\right\|_{V_{2}}^{2}=-\lambda_{0,2}\left\|u_{0,2}\right\|_{L^{2}}^{2}+\int_{\mathbf{R}^{N}} \mu_{2} u_{0,2}^{2 p_{2}+2}+\beta u_{0,1}^{p_{3}+1} u_{0,2}^{p_{3}+1} \mathrm{~d} x, \\
\left\|\nabla w_{0,1}\right\|_{L^{2}}^{2}=-\lambda_{0,1}\left\|w_{0,1}\right\|_{L^{2}}^{2}+\int_{\mathbf{R}^{N}} \mu_{1} w_{0,1}^{2 p_{1}+2}+\beta w_{0,1}^{p_{3}+1} w_{0,2}^{p_{3}+1} \mathrm{~d} x, \\
\left\|\nabla w_{0,2}\right\|_{L^{2}}^{2}=-\lambda_{0,2}\left\|w_{0,2}\right\|_{L^{2}}^{2}+\int_{\mathbf{R}^{N}} \mu_{2} w_{0,2}^{2 p_{2}+2}+\beta w_{0,1}^{p_{3}+1} w_{0,2}^{p_{3}+1} \mathrm{~d} x .
\end{gathered}
$$

Recalling $\alpha_{i}=\left\|u_{0, i}\right\|_{L^{2}}^{2}+\left\|w_{0, i}\right\|_{L^{2}}^{2}$, we have

$$
\left\|u_{0, i}\right\|_{V_{i}}^{2}+\left\|\nabla w_{0, i}\right\|_{L^{2}}^{2}=-\lambda_{0, i} \alpha_{i}+\mu_{i} \int_{\mathbf{R}^{N}} u_{0, i}^{2 p_{i}+2}+w_{0, i}^{2 p_{i}+2} \mathrm{~d} x+\beta \int_{\mathbf{R}^{N}} u_{0,1}^{p_{3}+1} u_{0,2}^{p_{3}+1}+w_{0,1}^{p_{3}+1} w_{0,2}^{p_{3}+1} \mathrm{~d} x .
$$

Thus,

$$
\begin{aligned}
& E\left(\tau_{n, 1}\left[u_{0,1}+w_{n, 1}\right], \tau_{n, 2}\left[u_{0,2}+w_{n, 2}\right]\right) \\
& \leq E\left(u_{0}\right)+E_{\infty}\left(w_{0}\right)-\int_{\mathbf{R}^{N}} \mu_{1} u_{0,1} w_{n, 1}^{2 p_{1}+1}+\mu_{2} u_{0,2} w_{n, 2}^{2 p_{2}+1}+\beta u_{0,2} w_{n, 1}^{p_{3}+1} w_{n, 2}^{p_{3}} \mathrm{~d} x \\
& \quad+ \begin{cases}o\left(\kappa_{n, 1}^{\theta_{0}}\right) & \text { if } \kappa_{n, 1}>0, \\
o\left(\kappa_{n, 2}^{\theta_{0}}\right) & \text { if } \kappa_{n, 1}=0<\kappa_{n, 2} .\end{cases}
\end{aligned}
$$

\section{Step 4: Conclusion}

Recalling (3.36), we first consider the case $\kappa_{n, 1}>0$. In this case, by Lemma 3.1, we notice that

$$
c_{0}(1+|x|)^{-(N-1) / 2} \exp \left(-\sqrt{\lambda_{0,1}}|x|\right) \leq u_{0,1}(x), \quad w_{n, 1}^{2 p_{1}+1}(x) \leq \exp \left(-\sqrt{\lambda_{0,1}+\eta_{0}}|x|\right)
$$

for some $\eta_{0}>0$. Hence, Lemma 3.3 yields

$$
\int_{\mathbf{R}^{N}} u_{0,1} w_{n, 1}^{2 p_{1}+1} \mathrm{~d} x \geq c(1+n)^{-(N-1) / 2} \exp \left(-\sqrt{\lambda_{0,1}} n\right) .
$$

Since $\theta_{0}>1$, it follows from Step 2 that

$$
\frac{\kappa_{n, 1}^{\theta_{0}}}{(1+n)^{-(N-1) / 2} \exp \left(-\sqrt{\lambda_{0,1}} n\right)} \rightarrow 0 \quad \text { as } n \rightarrow \infty .
$$

Thus by (3.52), for sufficiently large $n$, we obtain

$$
E\left(\tau_{n, 1}\left[u_{0,1}+w_{n, 1}\right], \tau_{n, 2}\left[u_{0,2}+w_{n, 2}\right]\right)<E\left(u_{0}\right)+E_{\infty}\left(w_{0}\right)=e\left(\gamma_{u_{0}}\right)+e_{\infty}\left(\gamma_{w_{0}}\right) .
$$

Hence, (3.35) holds.

Next, suppose $\kappa_{n, 1}=0<\kappa_{n, 2}$. Since $w_{n, 1} u_{0,1} \equiv 0,(3.52)$ becomes

$$
E\left(u_{0,1}+w_{n, 1}, \tau_{n, 2}\left[u_{0,2}+w_{n, 2}\right]\right) \leq E\left(u_{0}\right)+E_{\infty}\left(w_{0}\right)-\int_{\mathbf{R}^{N}} \mu_{2} u_{0,2} w_{n, 2}^{2 p_{2}+1}+\beta u_{0,2} w_{n, 1}^{p_{3}+1} w_{n, 2}^{p_{3}} \mathrm{~d} x+o\left(\kappa_{n, 2}^{\theta_{0}}\right) .
$$

We first treat the case $u_{0,1}>0$ and $w_{0,1} \equiv 0$, hence, we have

$$
E\left(u_{0,1}+w_{n, 1}, \tau_{n, 2}\left[u_{0,2}+w_{n, 2}\right]\right) \leq E\left(u_{0}\right)+E_{\infty}\left(w_{0}\right)-\int_{\mathbf{R}^{N}} \mu_{2} u_{0,2} w_{n, 2}^{2 p_{2}+1} \mathrm{~d} x+o\left(\kappa_{n, 2}^{\theta_{0}}\right) .
$$

When either $1 \leq p_{3}$ or $0<p_{3}<1$ and $\lambda_{0,3}=\left(1+p_{3}\right)^{2} \lambda_{0,1} /\left(1-p_{3}\right)^{2}>\lambda_{0,2}$, it follows from Lemmas 3.1 and 3.3 that for some $c_{1}, c_{2}>0$,

$$
\begin{aligned}
\int_{\mathbf{R}^{N}} u_{0,2} w_{n, 2}^{2 p_{2}+1} \mathrm{~d} x & \geq \int_{\mathbf{R}^{N}} c_{1} w_{n, 2}^{2 p_{2}+1}(1+|x|)^{-(N-1) / 2} \exp \left(-\sqrt{\lambda_{0,2}}|x|\right) \mathrm{d} x \\
& \geq c_{2} n^{-(N-1) / 2} \exp \left(-\sqrt{\lambda_{0,2}} n\right) .
\end{aligned}
$$


On the other hand, by Step 2 and $\theta_{0}>1$, we have

$$
\frac{\kappa_{n, 2}^{\theta_{0}}}{n^{-(N-1) / 2} \exp \left(-\sqrt{\lambda_{0,2}} n\right)} \rightarrow 0 \quad \text { as } n \rightarrow \infty .
$$

From (3.53), we observe that (3.35) holds.

Next suppose $0<p_{3}<1$ and $\lambda_{0,3} \leq \lambda_{0,2}$. In this case, Lemmas 3.1 and 3.3 imply that for every $\lambda_{0,3}<\lambda_{2}$

$$
\int_{\mathbf{R}^{N}} u_{0,2} w_{n, 2}^{2 p_{2}+1} \mathrm{~d} x \geq C_{\lambda_{2}} \exp \left(-\sqrt{\lambda_{2}} n\right) .
$$

Noting $\theta_{0}>1$ and Step 2, we see that

$$
\kappa_{n, 2}^{\theta_{0}}\left(\int_{\mathbf{R}^{N}} u_{0,2} w_{n, 2}^{2 p_{2}+1} \mathrm{~d} x\right)^{-1} \rightarrow 0 \quad \text { as } n \rightarrow \infty
$$

which gives (3.35) through (3.53).

Finally, we treat the case $u_{0,1} \equiv 0$ and $0<u_{0,2}, w_{0,1}, w_{0,2}$. In this case, (3.52) becomes

$$
E\left(w_{n, 1}, \tau_{n, 2}\left[u_{0,2}+w_{n, 2}\right]\right) \leq E\left(u_{0}\right)+E_{\infty}\left(w_{0}\right)-\int_{\mathbf{R}^{N}} \mu_{2} u_{0,2} w_{n, 2}^{2 p_{2}+1}+\beta u_{0,2} w_{n, 1}^{p_{3}+1} w_{n, 2}^{p_{3}} \mathrm{~d} x+o\left(\kappa_{n, 2}^{\theta_{0}}\right) .
$$

When either $1 \leq p_{3}$ or else $0<p_{3}<1$ and $\lambda_{0,3}>\lambda_{0,2}$, we can use a similar argument and obtain

$$
\kappa_{n, 2}^{\theta_{0}}\left(\int_{\mathbf{R}^{N}} u_{0,2} w_{n, 2}^{2 p_{2}+1} \mathrm{~d} x\right)^{-1} \rightarrow 0
$$

and (3.35) holds.

On the other hand, when $0<p_{3}<1$ and $\lambda_{0,3} \leq \lambda_{0,2}$, by (3.1) and (3.3) with $u_{0,1} \equiv 0$, one has

$$
\begin{aligned}
\int_{\mathbf{R}^{N}} \mu_{2} u_{0,2} w_{n, 2}^{2 p_{2}+1}+\beta u_{0,2} w_{n, 1}^{p_{3}+1} w_{n, 2}^{p_{3}} \mathrm{~d} x & =\int_{\mathbf{R}^{N}} \nabla w_{n, 2} \cdot \nabla u_{0,2}+\lambda_{0,2} w_{n, 2} u_{0,2} \mathrm{~d} x \\
& =\int_{\mathbf{R}^{N}}-V_{2}(x) u_{0,2} w_{n, 2}+\mu_{2} u_{0,2}^{2 p_{2}+1} w_{n, 2} \mathrm{~d} x \\
& \geq \mu_{2} \int_{\mathbf{R}^{N}} u_{0,2}^{2 p_{2}+1} w_{n, 2} \mathrm{~d} x .
\end{aligned}
$$

Hence, by Lemmas 3.1 and 3.3, for every $\lambda_{1}<\lambda_{0,3}<\lambda_{2}$, there exist $C_{\lambda_{1}}, C_{\lambda_{2}}>0$ such that

$$
C_{\lambda_{2}} \exp \left(-\sqrt{\lambda_{2}} n\right) \leq \int_{\mathbf{R}^{N}} u_{0,2}^{2 p_{2}+1} w_{n, 2} \mathrm{~d} x \leq C_{\lambda_{1}} \exp \left(-\sqrt{\lambda_{1}} n\right) .
$$

Thus, Step 2 yields

$$
\kappa_{n, 2}^{\theta_{0}}\left(\int_{\mathbf{R}^{N}} u_{0,2}^{2 p_{2}+1} w_{n, 2} \mathrm{~d} x\right)^{-1} \rightarrow 0 \quad \text { as } n \rightarrow \infty
$$

and (3.35) holds.

Since (3.35) gives a contradiction, the dichotomy does not happen and compactness occurs.

\section{Proof of Theorem 1.2}

In this section, we shall prove Theorem 1.2 and hereafter we always suppose (p1) and (V2). Let $\left(u_{n}\right) \subset \widetilde{M}(\alpha)$ be any minimizing sequence for $\widetilde{e}(\alpha)$. From Lemmas 2.1, 2.2 and 2.3, we may suppose that $\left(u_{n}\right)$ is bounded in $\widetilde{H}$ and satisfies

$$
\left\|E^{\prime}\left(u_{n}\right)-\lambda_{n, 1} Q_{1}^{\prime}\left(u_{n}\right)-\lambda_{n, 2} Q_{2}^{\prime}\left(u_{n}\right)\right\|_{(\widetilde{H})^{*}} \rightarrow 0, \quad\left\|\left(u_{n, i}\right)_{-}\right\|_{L^{2}} \rightarrow 0 .
$$

Furthermore, let $u_{n} \rightarrow u_{0}$ weakly in $\widetilde{H}$ and $\lambda_{n, i} \rightarrow \lambda_{0, i}$. By $V_{2}(x) \rightarrow \infty$ as $|x| \rightarrow \infty$ in (V2) and the result in [5], one sees

$$
\left\|u_{n, 2}-u_{0,2}\right\|_{L^{q}} \rightarrow 0 \text { for all } q \in\left[2,2^{*}\right) .
$$

Hence, $\alpha_{2}=\left\|u_{0,2}\right\|_{L^{2}}^{2}$. Thus, what remains to prove is $\alpha_{1}=\left\|u_{0,1}\right\|_{L^{2}}^{2}$ since this leads to $\left\|u_{n, 1}-u_{0,1}\right\|_{L^{2}} \rightarrow$ 0 and $\left\|u_{n}-u_{0}\right\|_{\widetilde{H}} \rightarrow 0$ from $E\left(u_{n}\right) \rightarrow \widetilde{e}(\alpha)$. 
We argue indirectly and suppose

$$
\left\|u_{0,1}\right\|_{L^{2}}^{2}<\alpha_{1} .
$$

We first show

Lemma 4.1. $\lim _{n \rightarrow \infty} \sup _{z \in \mathbf{Z}^{N}}\left\|u_{n, 1}-u_{0,1}\right\|_{L^{2}(z+Q)}>0$.

Proof. If $\sup _{z \in \mathbf{Z}^{N}}\left\|u_{n, 1}-u_{0,1}\right\|_{L^{2}(z+Q)} \rightarrow 0$, then we have $\left\|u_{n, 1}-u_{0,1}\right\|_{L^{q}} \rightarrow 0$ for any $2<q<2^{*}$. Therefore, the weak lower semicontinuity of the norms and (4.2) yield

$$
\widetilde{e}\left(\left\|u_{0,1}\right\|_{L^{2}}^{2}, \alpha_{2}\right) \leq E\left(u_{0}\right) \leq \liminf _{n \rightarrow \infty} E\left(u_{n}\right)=\widetilde{e}(\alpha) .
$$

Next, remark that $e_{1}(\gamma)<0$ holds for any $\gamma>0$. See [14,40]. Thus, Lemma 2.1 (iii) and (4.3) give a contradiction:

$$
\widetilde{e}\left(\left\|u_{0,1}\right\|_{L^{2}}^{2}, \alpha_{2}\right) \leq \widetilde{e}(\alpha) \leq \widetilde{e}\left(\left\|u_{0,1}\right\|_{L^{2}}^{2}, \alpha_{2}\right)+e_{1}\left(\alpha_{1}-\left\|u_{0,1}\right\|_{L^{2}}^{2}\right)<\widetilde{e}\left(\left\|u_{0,1}\right\|_{L^{2}}^{2}, \alpha_{2}\right) .
$$

Hence, Lemma 4.1 holds.

From Lemma 4.1, we may find $\left(y_{n}\right)_{n=1}^{\infty} \subset \mathbf{R}^{N}$ such that

$$
\left|y_{n}\right| \rightarrow \infty, \quad u_{n, 1}\left(\cdot+y_{n}\right) \rightarrow w_{0,1} \not \equiv 0 \quad \text { weakly in } H^{1}\left(\mathbf{R}^{N}\right) .
$$

Next, as in Lemma 3.6, we shall prove

Lemma 4.2. We have $\left\|u_{n, 1}-u_{0,1}-w_{0,1}\left(\cdot-y_{n}\right)\right\|_{H^{1}} \rightarrow 0$ and

$$
\widetilde{e}(\alpha)=\widetilde{e}\left(\left\|u_{0,1}\right\|_{L^{2}}^{2}, \alpha_{2}\right)+e_{1}\left(\left\|w_{0,1}\right\|_{L^{2}}^{2}\right)=E\left(u_{0}\right)+E_{1}\left(w_{0,1}\right) .
$$

Proof. Since a proof is similar to that of Lemma 3.6, we only give a sketch of the proof. We first suppose that $\lim _{n \rightarrow \infty}\left\|u_{n, 1}-u_{0,1}-w_{0,1}\left(\cdot-y_{n}\right)\right\|_{L^{2}}=\delta_{0}>0$. By $V_{1}(x) \rightarrow 0$ as $|x| \rightarrow \infty$ due to (V2), we may prove

$$
\begin{aligned}
& \left.E\left(u_{n}\right)-E\left(u_{0}\right)-E\left(w_{0,1}\left(\cdot-y_{n}\right), 0\right)-E\left(u_{n, 1}-u_{0,1}-w_{0,1}\left(\cdot-y_{n}\right)\right), 0\right) \rightarrow 0, \\
& 0<\delta_{0}=\alpha_{1}-\left\|u_{0,1}\right\|_{L^{2}}^{2}-\left\|w_{0,1}\right\|_{L^{2}}^{2} .
\end{aligned}
$$

From $\left|y_{n}\right| \rightarrow \infty$ and $u_{n, 1} \rightarrow u_{0,1}$ in $L_{\mathrm{loc}}^{q}\left(\mathbf{R}^{N}\right)$ for $1 \leq q<2^{*}$, it follows that

$$
\begin{aligned}
& \liminf _{n \rightarrow \infty} E\left(w_{0,1}\left(\cdot-y_{n}\right), 0\right)=\liminf _{n \rightarrow \infty} E_{1}\left(w_{0,1}\left(\cdot-y_{n}\right)\right) \geq e_{1}\left(\left\|w_{0,1}\right\|_{L^{2}}^{2}\right), \\
& \left.\liminf _{n \rightarrow \infty} E\left(u_{n, 1}-u_{0,1}-w_{0,1}\left(\cdot-y_{n}\right)\right), 0\right)=\liminf _{n \rightarrow \infty} E_{1}\left(u_{n, 1}-u_{0,1}-w_{0,1}\left(\cdot-y_{n}\right)\right) \geq e_{1}\left(\delta_{0}\right) .
\end{aligned}
$$

Hence, we obtain

$$
\widetilde{e}(\alpha) \geq \widetilde{e}\left(\left\|u_{0,1}\right\|_{L^{2}}^{2}, \alpha_{2}\right)+e_{1}\left(\left\|w_{0,1}\right\|_{L^{2}}^{2}\right)+e_{1}\left(\delta_{0}\right) .
$$

For $e_{1}(\alpha)$, it is known that $e_{1}(\gamma+\delta)<e_{1}(\gamma)+e_{1}(\delta)$ (see $[14,40]$ ). Combining this with Lemma 2.1 (iii), we obtain a contradiction:

$$
\widetilde{e}\left(\left\|u_{0,1}\right\|_{L^{2}}^{2}, \alpha_{2}\right)+e_{1}\left(\left\|w_{0,1}\right\|_{L^{2}}^{2}+\delta_{0}\right)<\widetilde{e}(\alpha) \leq \widetilde{e}\left(\left\|u_{0,1}\right\|_{L^{2}}^{2}, \alpha_{2}\right)+e_{1}\left(\left\|w_{0,1}\right\|_{L^{2}}^{2}+\delta_{0}\right) .
$$

Therefore, $\left\|u_{n, 1}-u_{0,1}-w_{0,1}\left(\cdot-y_{n}\right)\right\|_{L^{2}} \rightarrow 0$.

The rest of the proof is identical to that of Lemma 3.6 and we omit the detail.

To proceed, by (4.1) and $\left\|u_{n, 2}-u_{0,2}\right\|_{L^{2}} \rightarrow 0$, we observe that $u_{0}$ satisfies (3.1) and $w_{0,1}$ is a positive solution of

$$
-\Delta w_{0,1}+\lambda_{0,1} w_{0,1}=\mu_{1} w_{0,1}^{2 p_{2}+1} \quad \text { in } \mathbf{R}^{N}
$$

Next we show $\lambda_{0,1}>0$.

Lemma 4.3. We have $\lambda_{0,1}>0$. 
Proof. Since $w_{0,1} \in H^{1}\left(\mathbf{R}^{N}\right)$ is a positive solution of (4.5) with $2 p_{1}+1<2^{*}-1$, as in [6], when $N \geq 2$, we may show that $w_{0,1}$ satisfies the Pohozaev identity

$$
0=\frac{N-2}{2 N}\left\|\nabla w_{0,1}\right\|_{L^{2}}^{2}-\frac{\mu_{1}}{2 p_{2}+2}\left\|w_{0,1}\right\|_{L^{2 p_{1}+2}}^{2 p_{1}+2}+\frac{\lambda_{0,1}}{2}\left\|w_{0,1}\right\|_{L^{2}}^{2} .
$$

We note that when $N=1,(4.6)$ also holds since $w_{0,1}$ satisfies

$$
\frac{1}{2}\left(w_{0,1}^{\prime}(x)\right)^{2}+\frac{\mu_{1}}{2 p_{1}+2} w_{0,1}^{2 p_{1}+2}(x)-\frac{\lambda_{0,1}}{2} w_{0,1}^{2}(x) \equiv 0 \quad \text { in } \mathbf{R} .
$$

Thus, from $\left\|\nabla w_{0,1}\right\|_{L^{2}}^{2}+\lambda_{0,1}\left\|w_{0,1}\right\|_{L^{2}}^{2}=\mu_{1}\left\|w_{0,1}\right\|_{L^{2 p_{1}+2}}^{2 p_{1}+2}$, it follows that

$$
0=\left(\frac{N-2}{2 N}-\frac{1}{2 p_{1}+2}\right)\left\|\nabla w_{0,1}\right\|_{L^{2}}^{2}+\left(\frac{1}{2}-\frac{1}{2 p_{1}+2}\right) \lambda_{0,1}\left\|w_{0,1}\right\|_{L^{2}}^{2},
$$

which implies $\lambda_{0,1}>0$ due to $w_{0,1} \not \equiv 0$.

By (V2), especially $V_{2}(x) \rightarrow \infty$ as $|x| \rightarrow \infty, \lambda_{0,1}>0$ and (3.1), as in the proof of Lemma 3.1 (Steps 1 and 2) and [19], if $u_{0,1} \not \equiv 0$, then for each $\lambda \in\left(0, \lambda_{0,1}\right)$ we may find a $C_{\lambda}>0$ such that for all $x \in \mathbf{R}^{N}$,

$$
\begin{aligned}
& u_{0,1}(x)+u_{0,2}(x) \leq C_{\lambda} \exp (-\sqrt{\lambda}|x|), \\
& c_{0}(1+|x|)^{-(N-1) / 2} \exp \left(-\sqrt{\lambda_{0,1}}|x|\right) \leq u_{0,1}(x), \\
& c_{0}(1+|x|)^{-(N-1) / 2} \exp \left(-\sqrt{\lambda_{0,1}}|x|\right) \leq w_{0,1}(x) \leq c_{1}(1+|x|)^{-(N-1) / 2} \exp \left(-\sqrt{\lambda_{0,1}}|x|\right)
\end{aligned}
$$

where $0<c_{0} \leq c_{1}$. Now we derive a contradiction and complete the proof of Theorem 1.2:

Proof of Theorem 1.2. As in the proof of Theorem 1.1, we set

$$
w_{n}(x):=w_{0,1}\left(x-n \mathbf{e}_{1}\right), \quad \tau_{n}:=\frac{\sqrt{\alpha_{1}}}{\left\|u_{0,1}+w_{n}\right\|_{L^{2}}}, \quad \kappa_{n}:=\left\langle u_{0,1}, w_{n}\right\rangle_{L^{2}} .
$$

Then

$$
\kappa_{n} \rightarrow 0, \quad \tau_{n}=1-\frac{\kappa_{n}}{\alpha_{1}}+O\left(\kappa_{n}^{2}\right) .
$$

Since $\left(\tau_{n}\left(u_{0,1}+w_{n}\right), u_{0,2}\right) \in \widetilde{M}(\alpha)$, it follows that

$$
\begin{aligned}
\widetilde{e}(\alpha) \leq & E\left(\tau_{n}\left(u_{0,1}+w_{n}\right), u_{0,2}\right) \\
= & \frac{\tau_{n}^{2}}{2}\left\|u_{0,1}+w_{n}\right\|_{V_{1}}^{2}+\frac{1}{2}\left\|u_{0,2}\right\|_{V_{2}}^{2}-\frac{\mu_{1} \tau_{n}^{2 p_{1}+2}}{2 p_{1}+2} \int_{\mathbf{R}^{N}}\left(u_{0,1}+w_{n}\right)^{2 p_{1}+2} \mathrm{~d} x-\frac{\mu_{2}}{2 p_{2}+2} \int_{\mathbf{R}^{N}} u_{0,2}^{2 p_{2}+2} \mathrm{~d} x \\
& -\frac{\beta \tau_{n}^{p_{3}+1}}{p_{3}+1} \int_{\mathbf{R}^{N}}\left(u_{0,1}+w_{n}\right)^{p_{3}+1} u_{0,2}^{p_{3}+1} \mathrm{~d} x \\
= & \frac{1}{2}\left(1-\frac{2 \kappa_{n}}{\alpha_{1}}\right)\left(\left\|u_{0,1}\right\|_{V_{1}}^{2}+2\left\langle u_{0,1}, w_{n}\right\rangle_{V_{1}}+\left\|w_{n}\right\|_{V_{1}}^{2}\right)+\frac{1}{2}\left\|u_{0,2}\right\|_{V_{2}}^{2}-\frac{\mu_{2}}{2 p_{2}+2} \int_{\mathbf{R}^{N}} u_{0,2}^{2 p_{2}+2} \mathrm{~d} x \\
& -\frac{\mu_{1}}{2 p_{1}+2}\left(1-\frac{2 p_{1}+2}{\alpha_{1}} \kappa_{n}\right) \int_{\mathbf{R}^{N}}\left(u_{0,1}+w_{n}\right)^{2 p_{1}+2} \mathrm{~d} x \\
& -\frac{\beta}{p_{3}+1}\left(1-\frac{p_{3}+1}{\alpha_{1}} \kappa_{n}\right) \int_{\mathbf{R}^{N}}\left(u_{0,1}+w_{n}\right)^{p_{3}+1} u_{0,2}^{p_{3}+1} \mathrm{~d} x+O\left(\kappa_{n}^{2}\right) .
\end{aligned}
$$

When $u_{0,1} \equiv 0$, we have $\kappa_{n}=0$. Since $u_{0,2}>0$ and $w_{n}>0$, (4.8) becomes $\widetilde{e}(\alpha)$

$\leq \frac{1}{2}\left\|w_{n}\right\|_{V_{1}}^{2}+\frac{1}{2}\left\|u_{0,2}\right\|_{V_{2}}^{2}-\frac{\mu_{2}}{2 p_{2}+2} \int_{\mathbf{R}^{N}} u_{0,2}^{2 p_{2}+2} \mathrm{~d} x-\frac{\mu_{1}}{2 p_{1}+2} \int_{\mathbf{R}^{N}} w_{n}^{2 p_{1}+2} \mathrm{~d} x-\frac{\beta}{p_{3}+1} \int_{\mathbf{R}^{N}} w_{n}^{p_{3}+1} u_{0,2}^{p_{3}+1} \mathrm{~d} x$ $<E\left(u_{0}\right)+E_{1}\left(w_{0,1}\right)$.

Hence, this contradicts (4.4). 
Next, we suppose $u_{0,1} \not \equiv 0$ and it follows from $u_{0,1}, w_{n}>0,(4.7)$ and (4.5) that

$$
\begin{aligned}
\left\langle u_{0,1}, w_{n}\right\rangle_{V_{1}} & =-\lambda_{0,1} \kappa_{n}+\int_{\mathbf{R}^{N}} \mu_{1} u_{0,1}^{2 p_{1}+1} w_{n}+\beta u_{0,1}^{p_{3}} u_{0,2}^{p_{3}+1} w_{n} \mathrm{~d} x=O\left(\kappa_{n}^{1 / 2}\right), \\
\left\|u_{0,1}\right\|_{V_{1}}^{2} & =-\lambda_{0,1}\left\|u_{0,1}\right\|_{L^{2}}^{2}+\int_{\mathbf{R}^{N}} \mu_{1} u_{0,1}^{2 p_{1}+2}+\beta u_{0,1}^{p_{3}+1} u_{0,2}^{p_{3}+1} \mathrm{~d} x, \\
\left\|\nabla w_{n}\right\|_{L^{2}}^{2} & =-\lambda_{0,1}\left\|w_{n}\right\|_{L^{2}}^{2}+\int_{\mathbf{R}^{N}} \mu_{1} w_{n}^{2 p_{1}+2} \mathrm{~d} x .
\end{aligned}
$$

Therefore, by $V_{1}(x) \leq 0$, we obtain

$$
\begin{aligned}
& \frac{1}{2}\left(1-\frac{2 \kappa_{n}}{\alpha_{1}}\right)\left(\left\|u_{0,1}\right\|_{V_{1}}^{2}+2\left\langle u_{0,1}, w_{n}\right\rangle_{V_{1}}+\left\|w_{n}\right\|_{V_{1}}^{2}\right) \\
\leq & \frac{1}{2}\left(\left\|u_{0,1}\right\|_{V_{1}}^{2}+\left\|\nabla w_{0,1}\right\|_{L^{2}}^{2}\right)+\left\langle u_{0,1}, w_{n}\right\rangle_{V_{1}}-\frac{\kappa_{n}}{\alpha_{1}}\left(\left\|u_{0,1}\right\|_{V_{1}}^{2}+\left\|\nabla w_{0,1}\right\|_{L^{2}}^{2}\right)+O\left(\kappa_{n}^{3 / 2}\right) \\
= & \frac{1}{2}\left(\left\|u_{0,1}\right\|_{V_{1}}^{2}+\left\|\nabla w_{0,1}\right\|_{L^{2}}^{2}\right)+\mu_{1} \int_{\mathbf{R}^{N}} u_{0,1}^{2 p_{1}+1} w_{n} \mathrm{~d} x+\beta \int_{\mathbf{R}^{N}} u_{0,1}^{p_{3}} u_{0,2}^{p_{3}+1} w_{n} \mathrm{~d} x \\
& \quad-\frac{\kappa_{n}}{\alpha_{1}}\left(\lambda_{0,1} \alpha_{1}+\left\|u_{0,1}\right\|_{V_{1}}^{2}+\left\|\nabla w_{0,1}\right\|_{L^{2}}^{2}\right)+O\left(\kappa_{n}^{3 / 2}\right) .
\end{aligned}
$$

By Lemmas 3.3 and 3.4, (4.7) and $\kappa_{n} \rightarrow 0$, we observe that

$$
\begin{aligned}
- & \frac{\mu_{1}}{2 p_{1}+2}\left(1-\frac{2 p_{1}+2}{\alpha_{1}} \kappa_{n}\right) \int_{\mathbf{R}^{N}}\left(u_{0,1}+w_{n}\right)^{2 p_{1}+2} \mathrm{~d} x \\
\leq- & \frac{\mu_{1}}{2 p_{1}+2} \int_{\mathbf{R}^{N}} u_{0,1}^{2 p_{1}+2}+w_{n}^{2 p_{1}+2}+\left(2 p_{1}+2\right)\left(u_{0,1}^{2 p_{1}+1} w_{n}+w_{n}^{2 p_{1}+1} u_{0,1}\right) \mathrm{d} x \\
& +\frac{\kappa_{n}}{\alpha_{1}} \mu_{1} \int_{\mathbf{R}^{N}} u_{0,1}^{2 p_{1}+2}+w_{n}^{2 p_{1}+2} \mathrm{~d} x+O\left(\kappa_{n}^{\theta}\right)
\end{aligned}
$$

for some $\theta>1$. Next, by

$$
a^{p_{3}+1}+\left(p_{3}+1\right) a^{p_{3}} b \leq(a+b)^{p_{3}+1} \leq a^{p_{3}+1}+\left(p_{3}+1\right)(a+b)^{p_{3}} b \text { for each } a, b \geq 0,
$$

we obtain

$$
\begin{aligned}
& -\frac{\beta}{p_{3}+1} \int_{\mathbf{R}^{N}}\left(u_{0,1}+w_{n}\right)^{p_{3}+1} u_{0,2}^{p_{3}+1} \mathrm{~d} x \\
\leq & -\frac{\beta}{p_{3}+1} \int_{\mathbf{R}^{N}} u_{0,1}^{p_{3}+1} u_{0,2}^{p_{3}+1}+\left(p_{3}+1\right) u_{0,1}^{p_{3}} u_{0,2}^{p_{3}+1} w_{n} \mathrm{~d} x
\end{aligned}
$$

and

$$
\frac{\kappa_{n}}{\alpha_{1}} \beta \int_{\mathbf{R}^{N}}\left(u_{0,1}+w_{n}\right)^{p_{3}+1} u_{0,2}^{p_{3}+1} \mathrm{~d} x \leq \frac{\kappa_{n}}{\alpha_{1}} \beta \int_{\mathbf{R}^{N}} u_{0,1}^{p_{3}+1} u_{0,2}^{p_{3}+1} \mathrm{~d} x+O\left(\kappa_{n}^{\theta}\right) .
$$

From (4.8)-(4.13) and $\alpha_{1}=\left\|u_{0,1}\right\|_{L^{2}}^{2}+\left\|w_{0,1}\right\|_{L^{2}}^{2}$, it follows that

$$
\begin{aligned}
\widetilde{e}(\alpha) \leq & \frac{1}{2}\left(\left\|u_{0,1}\right\|_{V_{1}}^{2}+\left\|\nabla w_{0,1}\right\|_{L^{2}}^{2}\right)+\mu_{1} \int_{\mathbf{R}^{N}} u_{0,1}^{2 p_{1}+1} w_{n} \mathrm{~d} x+\beta \int_{\mathbf{R}^{N}} u_{0,1}^{p_{3}} u_{0,2}^{p_{3}+1} w_{n} \mathrm{~d} x \\
& -\frac{\kappa_{n}}{\alpha_{1}}\left(\alpha_{1} \lambda_{0,1}+\left\|u_{0,1}\right\|_{V_{1}}^{2}+\left\|\nabla w_{0,1}\right\|_{L^{2}}^{2}\right)+\frac{1}{2}\left\|u_{0,2}\right\|_{V_{2}}^{2}-\frac{\mu_{2}}{2 p_{2}+2} \int_{\mathbf{R}^{N}} u_{0,2}^{2 p_{2}+2} \mathrm{~d} x \\
& -\frac{\mu_{1}}{2 p_{1}+2} \int_{\mathbf{R}^{N}} u_{0,1}^{2 p_{1}+2}+w_{n}^{2 p_{1}+2}+\left(2 p_{1}+2\right)\left(u_{0,1}^{2 p_{1}+1} w_{n}+w_{n}^{2 p_{1}+1} u_{0,1}\right) \mathrm{d} x \\
& +\frac{\kappa_{n}}{\alpha_{1}} \mu_{1} \int_{\mathbf{R}^{N}} u_{0,1}^{2 p_{1}+2}+w_{n}^{2 p_{1}+2} \mathrm{~d} x \\
& -\frac{\beta}{p_{3}+1} \int_{\mathbf{R}^{N}} u_{0,1}^{p_{3}+1} u_{0,2}^{p_{3}+1}+\left(p_{3}+1\right) u_{0,1}^{p_{3}} u_{0,2}^{p_{3}+1} w_{n} \mathrm{~d} x+\frac{\kappa_{n}}{\alpha_{1}} \beta \int_{\mathbf{R}^{N}} u_{0,1}^{p_{3}+1} u_{0,2}^{p_{3}+1} \mathrm{~d} x+O\left(\kappa_{n}^{\theta}\right) \\
= & E\left(u_{0}\right)+E_{1}\left(w_{0,1}\right)-\mu_{1} \int_{\mathbf{R}^{N}} w_{n}^{2 p_{1}+1} u_{0,1} \mathrm{~d} x+O\left(\kappa_{n}^{\theta}\right) .
\end{aligned}
$$


As in the proof of Theorem 1.1, since $\theta>1$, we see

$$
-\mu_{1} \int_{\mathbf{R}^{N}} w_{n}^{2 p_{1}+1} u_{0,1} \mathrm{~d} x+O\left(\kappa_{n}^{\theta}\right)<0
$$

for sufficiently large $n$, hence, (4.4) gives a contradiction:

$$
\widetilde{e}(\alpha)<E\left(u_{0}\right)+E_{1}\left(w_{0,1}\right)=\widetilde{e}(\alpha) .
$$

Thus, (4.3) may not happen and we get $\left\|u_{n, 1}-u_{0,1}\right\|_{L^{2}} \rightarrow 0$. Hence, Theorem 1.2 holds.

Acknowledgement. The first author was supported by JSPS KAKENHI Grant Number JP16K17623 and JP17H02851. The second author was supported by JSPS KAKENHI Grant Numbers 16K05225 and $17 \mathrm{KK} 0086$.

\section{Appendix A. Proof of (1.4) under $p_{3} \geq 1$}

In this appendix, following [28] (cf. [10,14]), we shall prove (1.4) under (p1), (V1) and $p_{3} \geq 1$. Here we allow the case $V_{1}(x) \equiv V_{2}(x) \equiv 0$ and in this case, we may assume $\theta \neq(0,0),(1,1)$ for $(1.4)$.

We begin with

Lemma A.1. Suppose (V1) and (p1).

(i) Let $\gamma=\left(\gamma_{1}, \gamma_{2}\right) \in\left[0, \alpha_{1}\right] \times\left[0, \alpha_{2}\right] \backslash\{(0,0)\}$. Then $e(\gamma) \leq e_{\infty}(\gamma)<0$.

(ii) Let $\gamma \in\left[0, \alpha_{1}\right] \times\left[0, \alpha_{2}\right] \backslash\{(0,0)\}$ with $\gamma_{i_{0}}>0$. Then there exists a $\delta_{\gamma}>0$ such that $\left\|u_{i_{0}}\right\|_{L^{2 p_{0}+2}}^{2 p_{i_{0}}+2} \geq$ $\delta_{\gamma}$ for all $u \in M(\gamma)$ with $E(u) \leq e(\gamma)+\delta_{\gamma}$ (resp. $\left.E_{\infty}(u) \leq e_{\infty}(\gamma)+\delta_{\gamma}\right)$.

(iii) The values $e\left(\alpha_{1}, 0\right), e\left(0, \alpha_{2}\right), e_{\infty}\left(\alpha_{1}, 0\right), e_{\infty}\left(0, \alpha_{2}\right)$ are attained by positive functions.

Proof. (i) See Lemma 2.1 and [21].

(ii) We only prove for $E$ and the case $\gamma_{1}>0$, and argue by contradiction. Let $\gamma_{1}>0$. If there exists $\left(u_{n}\right)_{n=1}^{\infty} \subset M(\gamma)$ such that $E\left(u_{n}\right) \rightarrow e(\gamma)$ and $\left\|u_{n, 1}\right\|_{L^{2 p_{1}+2}} \rightarrow 0$, then we obtain

$$
e(\gamma)=\lim _{n \rightarrow \infty} E\left(u_{n}\right) \geq \liminf _{n \rightarrow \infty} E\left(0, u_{n, 2}\right) \geq e\left(0, \gamma_{2}\right) .
$$

On the other hand, Lemma 2.1 and (i) give a contradiction:

$$
e(\gamma) \leq e\left(0, \gamma_{2}\right)+e_{\infty}\left(\gamma_{1}, 0\right)<e\left(0, \gamma_{2}\right) \leq e(\gamma)
$$

Thus, (ii) holds.

(iii) First, using the argument in [28, p.228-p.229] and [14, Chapter 8, section 3], we observe that the values $e\left(\alpha_{1}, 0\right), e\left(0, \alpha_{2}\right), e_{\infty}\left(\alpha_{1}, 0\right), e_{\infty}\left(0, \alpha_{2}\right)$ admit minimizers. Next, if $u$ is a minimizer of one of these values, then so is $|u|$. In addition, $|u|$ satisfies the elliptic equations and the strong maximum principle asserts $|u|>0$ in $\mathbf{R}^{N}$. Hence, $|u|$ is the desired minimizer.

For $i=1,2$, we set

$$
\begin{aligned}
\widetilde{E}_{i}(u) & :=\frac{1}{2}\left\|u_{i}\right\|_{V_{i}}^{2}-\frac{\mu_{i}}{2 p_{i}+2}\left\|u_{i}\right\|_{L^{2 p_{i}+2}}^{2 p_{i}+2}-\frac{\beta}{p_{3}+1}\left\|u_{1} u_{2}\right\|_{L^{p_{3}+1}}^{p_{3}+1}, \\
\widetilde{E}_{\infty, i}(u) & :=\frac{1}{2}\left\|\nabla u_{i}\right\|_{L^{2}}^{2}-\frac{\mu_{i}}{2 p_{i}+2}\left\|u_{i}\right\|_{L^{2 p_{i}+2}}^{2 p_{i}+2}-\frac{\beta}{p_{3}+1}\left\|u_{1} u_{2}\right\|_{L^{p_{3}+1}}^{p_{3}+1} .
\end{aligned}
$$

Then $\widetilde{E}_{i}(u) \leq \widetilde{E}_{\infty, i}(u), E(u)=\widetilde{E}_{1}(u)+E\left(0, u_{2}\right)=\widetilde{E}_{2}(u)+E\left(u_{1}, 0\right)$ and the same equalities hold for $E_{\infty}$ and $\widetilde{E}_{\infty, i}$.

Now fix $\theta=\left(\theta_{1}, \theta_{2}\right) \in[0,1] \times[0,1]$ with $\theta \neq(0,0)$ and assume $0<\theta_{1}<1$. Put $\gamma:=\left(\theta_{1} \alpha_{1}, \theta_{2} \alpha_{2}\right)$ and choose $u=\left(u_{1}, u_{2}\right), v=\left(v_{1}, v_{2}\right) \in\left(C_{0}^{\infty}\left(\mathbf{R}^{N}\right)\right)^{2}$ so that

$$
\left\|u_{i}\right\|_{L^{2}}^{2}=\theta_{i} \alpha_{i},\left\|v_{i}\right\|_{L^{2}}^{2}=\left(1-\theta_{i}\right) \alpha_{i}, E(u) \leq e(\gamma)+\delta_{\gamma}, E_{\infty}(v) \leq e_{\infty}(\alpha-\gamma)+\delta_{\alpha-\gamma}
$$


where $\delta_{\gamma}, \delta_{\alpha-\gamma}>0$ are constants in Lemma A.1(ii). Since $u$ and $v$ have compact support, we find an $n$ so that $u_{i}(x) v_{j}\left(x-n \mathbf{e}_{1}\right) \equiv 0$ for $i, j=1,2$ and write $v_{n, i}(x):=v_{i}\left(x-n \mathbf{e}_{1}\right)$. From Lemma A.1 (ii), $p_{3} \geq 1$ and $0<\theta_{1}<1$, it follows that

$$
\begin{aligned}
\theta_{1} e(\alpha) & \leq \theta_{1} E\left(\frac{u_{1}}{\sqrt{\theta_{1}}}, u_{2}+v_{n, 2}\right) \\
& =\theta_{1}\left(\widetilde{E}_{1}\left(\frac{u_{1}}{\sqrt{\theta_{1}}}, u_{2}+v_{n, 2}\right)+E\left(0, u_{2}+v_{n, 2}\right)\right) \\
& \leq \widetilde{E}_{1}(u)-\mu_{1} \frac{\theta_{1}^{-p_{1}}-1}{2 p_{1}+2}\left\|u_{1}\right\|_{L^{2 p_{1}+2}}^{2 p_{1}+2}-\beta \frac{\theta_{1}^{-\frac{p_{3}-1}{2}}-1}{p_{3}+1}\left\|u_{1} u_{2}\right\|_{L^{p_{3}+1}}^{p_{3}+1}+\theta_{1}\left(E\left(0, u_{2}\right)+E_{\infty}\left(0, v_{n, 2}\right)\right) \\
& \leq \widetilde{E}_{1}(u)-\mu_{1} \frac{\theta_{1}^{-p_{1}}-1}{2 p_{1}+2} \delta_{\gamma}+\theta_{1}\left(E\left(0, u_{2}\right)+E_{\infty}\left(0, v_{n, 2}\right)\right) .
\end{aligned}
$$

Similarly,

$$
\begin{aligned}
\left(1-\theta_{1}\right) e(\alpha) & \leq\left(1-\theta_{1}\right) E\left(\frac{v_{n, 1}}{\sqrt{1-\theta_{1}}}, u_{2}+v_{n, 2}\right) \\
& \leq \widetilde{E}_{\infty, 1}(v)-\mu_{1} \frac{\left(1-\theta_{1}\right)^{-p_{1}}-1}{2 p_{1}+2} \delta_{\alpha-\gamma}+\left(1-\theta_{1}\right)\left(E\left(0, u_{2}\right)+E_{\infty}\left(0, v_{n, 2}\right)\right) .
\end{aligned}
$$

Hence,

$$
\begin{aligned}
e(\alpha) & =\theta_{1} e(\alpha)+\left(1-\theta_{1}\right) e(\alpha) \\
& \leq E(u)+E_{\infty}(v)-\mu_{1} \frac{\theta_{1}^{-p_{1}}-1}{2 p_{1}+2} \delta_{\gamma}-\mu_{1} \frac{\left(1-\theta_{1}\right)^{-p_{1}}-1}{2 p_{1}+2} \delta_{\alpha-\gamma} .
\end{aligned}
$$

Since $u, v \in\left(C_{0}^{\infty}\left(\mathbf{R}^{N}\right)\right)^{2}$ are arbitrary elements satisfying (A.1), from $0<\theta_{1}<1$ we infer that

$$
\begin{aligned}
e(\alpha) & \leq e(\gamma)+e_{\infty}(\alpha-\gamma)-\mu_{1} \frac{\theta_{1}^{-p_{1}}-1}{2 p_{1}+2} \delta_{\gamma}-\mu_{1} \frac{\left(1-\theta_{1}\right)^{-p_{1}}-1}{2 p_{1}+2} \delta_{\alpha-\gamma} \\
& <e(\gamma)+e_{\infty}(\alpha-\gamma)=e\left(\theta_{1} \alpha_{1}, \theta_{2} \alpha_{2}\right)+e_{\infty}\left(\left(1-\theta_{1}\right) \alpha_{1},\left(1-\theta_{2}\right) \alpha_{2}\right) .
\end{aligned}
$$

Hence, (1.4) holds when $0<\theta_{1}<1$.

Remark that in a similar way, we may prove (1.4) when $0<\theta_{2}<1$. Thus, the remaining cases are $\theta=(1,0),(0,1),(1,1)$.

When $\theta=(1,0),(0,1)$, from Lemma A.1 (iii), to prove (1.4), we can argue as in Step 1 in the proof of Theorem 1.1. Therefore, in the case $V_{1}(x) \equiv V_{2}(x) \equiv 0,(1.4)$ holds and $e_{\infty}(\alpha)$ is attained.

Finally, let us consider the case $V_{1}(x) \not \equiv 0$ or $V_{2}(x) \not \equiv 0$. Notice that $e_{\infty}(\alpha)$ is attained and using a similar argument to Lemma A.1 (iii), we may choose a positive minimizer for $e_{\infty}(\alpha)$. Again, we may exploit the proof of Step 1 in the proof of Theorem 1.1 and conclude that (1.4) holds.

\section{REFERENCES}

[1] J. Albert and S. Bhattarai, Existence and stability of a two-parameter family of solitary waves for an NLS-KdV system. Adv. Differential Equations 18 (2013), no. 11-12, 1129-1164. 2

[2] A.H. Ardila, Orbital stability of standing waves for a system of nonlinear Schrödinger equations with three wave interaction. Nonlinear Anal. 167 (2018), 1-20. 2

[3] A. Bahri and Y.Y. Li, On a min-max procedure for the existence of a positive solution for certain scalar field equations in $\mathbf{R}^{N}$. Rev. Mat. Iberoamericana 6 (1990), no. 1-2, 1-15. 3, 6, 13

[4] A. Bahri and P.-L. Lions, On the existence of a positive solution of semilinear elliptic equations in unbounded domains. Ann. Inst. H. Poincaré Anal. Non Linéaire 14 (1997), no. 3, 365-413. 6

[5] T. Bartsch and Z.Q. Wang, Existence and multiplicity results for some superlinear elliptic problems on $\mathbf{R}^{N}$. Comm. Partial Differential Equations 20 (1995), no. 9-10, 1725-1741. 24

[6] H. Berestycki and P.-L. Lions, Nonlinear scalar field equations. I. Existence of a ground state. Arch. Rational Mech. Anal. 82 (1983), no. 4, 313-345. 26

[7] S. Bhattarai, Stability of solitary-wave solutions of coupled NLS equations with power-type nonlinearities. Adv. Nonlinear Anal. 4 (2015), no. 2, 73-90. 1 
[8] S. Bhattarai, Existence of travelling-wave solutions to a coupled system of Korteweg-de Vries equations. Nonlinear Anal. 127 (2015), 182-195. 2

[9] S. Bhattarai, Stability of normalized solitary waves for three coupled nonlinear Schrödinger equations. Discrete Contin. Dyn. Syst. 36 (2016), no. 4, 1789-1811. 2

[10] S. Bhattarai, On fractional Schrödinger systems of Choquard type. J. Differential Equations 263 (2017), no. 6, 31973229. 2, 3, 28

[11] S. Bhattarai, Existence and stability of standing waves for coupled nonlinear Hartree type equations. arXiv:1902.02618 [math.AP]. 2

[12] S. Bhattarai, A.J. Corcho and M. Panthee, Well-posedness for multicomponent Schrödinger-gKdV systems and stability of solitary waves with prescribed mass. J. Dynam. Differential Equations 30 (2018), no. 2, 845-881. 2

[13] H. Buljan, T. Schwartz, M. Segev, M. Soljačić, and D.N. Christodoulides, Polychromatic partially spatially incoherent solitons in a noninstantaneous Kerr nonlinear medium. J. Opt. Soc. Am. B Vol. 21 (2004), Issue 2, 397-404. 1

[14] T. Cazenave, Semilinear Schrödinger equations. Courant Lecture Notes in Mathematics, 10. New York University, Courant Institute of Mathematical Sciences, New York; American Mathematical Society, Providence, RI, 2003. xiv +323 pp. $3,25,28$

[15] D.N. Christodoulides, E.D. Eugenieva, T. H. Coskun, M. Segev and M. Mitchell, Equivalence of three approaches describing partially incoherent wave propagation in inertial nonlinear media. Phys. Rev. E 63 (2001), 035601(R). 1

[16] B.D. Esry, C.H. Greene, J.P. Burke, Jr. and J.L. Bohn, Hartree-Fock Theory for Double Condensates. Phys. Rev. Lett. 78 (1997), 3594-3597. 1

[17] T.-L. Ho and V.B. Shenoy, Binary Mixtures of Bose Condensates of Alkali Atoms. Phys. Rev. Lett. 77 (1996), 3276-3279. 1

[18] D. Garrisi, Orbitally stable standing-wave solutions to a coupled non-linear Klein-Gordon equation. Nonlinear dynamics in partial differential equations, 387-398, Adv. Stud. Pure Math., 64, Math. Soc. Japan, Tokyo, 2015. 2

[19] B. Gidas, W.M. Ni and L. Nirenberg, Symmetry of positive solutions of nonlinear elliptic equations in $\mathbf{R}^{n}$. Mathematical analysis and applications, Part A, pp. 369-402, Adv. in Math. Suppl. Stud., 7a, Academic Press, New York-London, 1981. 6, 9, 26

[20] T. Gou, Existence and orbital stability of standing waves to nonlinear Schrödinger system with partial confinement. J. Math. Phys. 59 (2018), no. 7, 071508, 12 pp. 1, 3

[21] T. Gou and L. Jeanjean, Existence and orbital stability of standing waves for nonlinear Schrödinger systems. Nonlinear Anal. 144 (2016), 10-22. 1, 3, 4, 17, 28

[22] Y. Guo, S. Li, J. Wei and X. Zeng, Ground states of two-component attractive Bose-Einstein condensates I: Existence and uniqueness. J. Funct. Anal. 276 (2019), no. 1, 183-230. 1

[23] Y. Guo, S. Li, J. Wei and X. Zeng, Ground states of two-component attractive Bose-Einstein condensates II: Semitrivial limit behavior. to appear in Trans. Amer. Math. Soc. 1

[24] N. Ikoma, Compactness of minimizing sequences in nonlinear Schrödinger systems under multiconstraint conditions. Adv. Nonlinear Stud. 14 (2014), no. 1, 115-136. 2, 3

[25] N. Ikoma and Y. Miyamoto, Stable standing waves of nonlinear Schrödinger equations with potentials and general nonlinearities. Preprint. 3

[26] E.H. Lieb and M. Loss, Analysis. Second edition. Graduate Studies in Mathematics 14. American Mathematical Society, Providence, RI, 2001. 4

[27] P.-L. Lions, The concentration-compactness principle in the calculus of variations. The locally compact case. I. Ann. Inst. H. Poincaré Anal. Non Linéaire 1 (1984), no. 2, 109-145. 2

[28] P.-L. Lions, The concentration-compactness principle in the calculus of variations. The locally compact case. II, Ann. Inst. H. Poincaré Anal. Non Linéaire 1 (1984), 223-283. 2, 3, 28

[29] J. Mawhin and M. Willem, Critical point theory and Hamiltonian systems. Applied Mathematical Sciences, 74. Springer-Verlag, New York, 1989. 5

[30] L. Maia, Liliane, E. Montefusco and B. Pellacci, Orbital stability property for coupled nonlinear Schrödinger equations. Adv. Nonlinear Stud. 10 (2010), no. 3, 681-705. 1

[31] E. Montefusco, B. Pellacci and M. Squassina, Soliton dynamics for CNLS systems with potentials. Asymptot. Anal. 66 (2010), no. 2, 61-86. 2

[32] N.V. Nguyen and Z.-Q. Wang, Orbital stability of solitary waves for a nonlinear Schrödinger system. Adv. Differential Equations 16 (2011), no. 9-10, 977-1000. 1

[33] N.V. Nguyen and Z.-Q. Wang, Orbital stability of solitary waves of a 3-coupled nonlinear Schrödinger system. Nonlinear Anal. 90 (2013), 1-26. 1

[34] B. Noris, H. Tavares and G. Verzini, Stable solitary waves with prescribed $L^{2}$-mass for the cubic Schrödinger system with trapping potentials. Discrete Contin. Dyn. Syst. 35 (2015), no. 12, 6085-6112. 1

[35] P. Öhberg and S. Stenholm, Hartree-Fock treatment of the two-component Bose-Einstein condensate. Phys. Rev. A 57 (1998), 1272-1279. 1 
[36] M. Ohta, Stability of solitary waves for coupled nonlinear Schrödinger equations. Nonlinear Anal. 26 (1996), no. 5, 933-939. 1

[37] M.H. Protter and H.F. Weinberger, Maximum Principles in Differential Equations. Corrected reprint of the 1967 original. Springer-Verlag, New York, 1984. 17

[38] H. Pu and N.P. Bigelow, Properties of Two-Species Bose Condensates. Phys. Rev. Lett. 80 (1998), 1130-1133. 1

[39] P. Quittner and P. Souplet, Superlinear parabolic problems. Blow-up, global existence and steady states. Birkhäuser Advanced Texts: Basler Lehrbücher. Birkhäuser Verlag, Basel, 2007. 17

[40] M. Shibata, Stable standing waves of nonlinear Schrödinger equations with a general nonlinear term, Manuscripta Math. 143 (2014), 221-237. 3, 25

[41] M. Shibata, A new rearrangement inequality and its application for $L^{2}$-constraint minimizing problems. Math. Z. 287 (2017), no. 1-2, 341-359. 3

[42] J. Wang and W. Yang, Normalized solutions and asymptotical behavior of minimizer for the coupled Hartree equations. J. Differential Equations 265 (2018), no. 2, 501-544. 2

[43] M. Willem, Minimax theorems. Progress in Nonlinear Differential Equations and their Applications, 24. Birkhäuser Boston, Inc., Boston, MA, 1996. 15, 16

Department of Mathematics, Faculty of Science and Technology, Keio University, Yagami Campus: 3-14-1 Hiyoshi, KohokU-Ku, Yokohama 223-8522, Japan

Email address: ikoma@math.keio.ac.jp

Graduate School of Mathematical Sciences, The University of Tokyo, 3-8-1 Komaba, Meguro-ku, Tokyo 153-8914, JAPAN

Email address: miyamoto@ms.u-tokyo.ac.jp 